\title{
Review \\ Requirements for Energy-Harvesting-Driven Edge Devices Using Task-Offloading Approaches
}

\author{
Meriam Ben Ammar 1,2,3,*(D), Imed Ben Dhaou ${ }^{4,5,6} \mathbb{D}^{\mathbb{D}}$, Dhouha El Houssaini ${ }^{1} \mathbb{D}$, Salwa Sahnoun ${ }^{2,3}$, \\ Ahmed Fakhfakh ${ }^{2,3}$ and Olfa Kanoun ${ }^{1}$ (D)
}

1 Measurements and Sensor Technology, Faculty of Electrical Engineering and Information Technology, Chemnitz University of Technology, W206, 09126 Chemnitz, Germany; dhouha.el-houssaini@etit.tu-chemnitz.de (D.E.H.); Olfa.Kanoun@etit.tu-chemnitz.de (O.K.)

2 National School of Electronics and Telecommunications of Sfax, University of Sfax, Sfax 3018, Tunisia; salwa.sahnoun@enetcom.usf.tn (S.S.); ahmed.fakhfakh@enetcom.usf.tn (A.F.)

3 Laboratory of Signals, Systems, Artificial Intelligence and Networks, Digital Research Center of Sfax, University of Sfax, Sfax 3038, Tunisia

4 Department of Computer Science, Hekma School of Engineering, Computing and Informatics, Dar Al-Hekma University, Jeddah 22246-4872, Saudi Arabia; imed.bendhaou@utu.fi

5 Department of Computing, University of Turku, FI-20014 Turku, Finland

6 Department of Technology, Higher Institute of Computer Sciences and Mathematics, University of Monastir, Monastir 5000, Tunisia

* Correspondence: meriam.ben-ammar@etit.tu-chemnitz.de

\section{check for} updates

Citation: Ben Ammar, M.; Ben Dhaou, I.; El Houssaini, D.; Sahnoun, S.; Fakhfakh, A.; Kanoun, O. Requirements for EnergyHarvesting-Driven Edge Devices Using Task-Offloading Approaches. Electronics 2022, 11, 383. https:// doi.org/10.3390/electronics11030383 Academic Editors: Shailendra Rajput, Moshe Averbukh and Noel Rodriguez

Received: 6 December 2021 Accepted: 19 January 2022 Published: 27 January 2022

Publisher's Note: MDPI stays neutral with regard to jurisdictional claims in published maps and institutional affiliations.

Copyright: (C) 2022 by the authors Licensee MDPI, Basel, Switzerland. This article is an open access article distributed under the terms and conditions of the Creative Commons Attribution (CC BY) license (https:// creativecommons.org/licenses/by/ $4.0 /)$.

\begin{abstract}
Energy limitations remain a key concern in the development of Internet of Medical Things (IoMT) devices since most of them have limited energy sources, mainly from batteries. Therefore, providing a sustainable and autonomous power supply is essential as it allows continuous energy sensing, flexible positioning, less human intervention, and easy maintenance. In the last few years, extensive investigations have been conducted to develop energy-autonomous systems for the IoMT by implementing energy-harvesting $(\mathrm{EH})$ technologies as a feasible and economically practical alternative to batteries. To this end, various EH-solutions have been developed for wearables to enhance power extraction efficiency, such as integrating resonant energy extraction circuits such as SSHI, S-SSHI, and P-SSHI connected to common energy-storage units to maintain a stable output for charge loads. These circuits enable an increase in the harvested power by $174 \%$ compared to the SEH circuit. Although IoMT devices are becoming increasingly powerful and more affordable, some tasks, such as machine-learning algorithms, still require intensive computational resources, leading to higher energy consumption. Offloading computing-intensive tasks from resource-limited user devices to resource-rich fog or cloud layers can effectively address these issues and manage energy consumption. Reinforcement learning, in particular, employs the Q-algorithm, which is an efficient technique for hardware implementation, as well as offloading tasks from wearables to edge devices. For example, the lowest reported power consumption using FPGA technology is $37 \mathrm{~mW}$. Furthermore, the communication cost from wearables to fog devices should not offset the energy savings gained from task migration. This paper provides a comprehensive review of joint energy-harvesting technologies and computation-offloading strategies for the IoMT. Moreover, power supply strategies for wearables, energy-storage techniques, and hardware implementation of the task migration were provided.
\end{abstract}

Keywords: energy harvesting; IoMT devices; energy autonomous; wearables; energy-storage; energy management; fog edge computing; task offloading; deep learning; reinforced learning; IoMT

\section{Introduction}

With the spread of Internet of Medical Things (IoMT) applications, more intelligent services are presently emerging in the healthcare and medical areas, such as remote patient monitoring [1,2], telemedicine [3], biometrics scanners [4] and vital signs monitoring [5,6]. 
In general, the IoMT comprises different and heterogeneous smart devices, such as wearables, wireless sensors, and medical monitors, which can be applied to the human body, at home or in hospitals to provide better and more efficient remote monitoring. By combining information technology with medical information, wearable devices can perform better monitoring of medical and healthcare applications, resulting in reduced complexity and enhanced efficiency. With the use of the IoMT, physicians and healthcare responsible are also able to access different and real-time medical databases, which ensures a better understanding and identification of their patients' health issues.

The IoMT presents an application of the Internet of Things (IoT) in the field of medical and healthcare. The IoT comprises physical network devices equipped with sensors, software, and network connections that facilitate data collection and transmission. It can integrate cloud services and fog centers, where complex and efficient data processing is carried out with high processing capabilities. Considering the basic concepts of the IoT, the general layer architecture of IoMT is illustrated in Figure 1. It comprises four main layers, namely the sensing layer, the edge layer, the fog layer and the cloud layer. In the sensing layer, the wireless sensors and medical devices are installed along with different actuators. They are responsible for sensing medical and physiological information, and executing specific controlling and monitoring requests such as laser positioning and equipment maintenance. The raw data collected from the end devices are collected and transmitted to the edge devices, where data processing, reduction and analysis are carried out. Devices with edge computing processors provide improved security while operating at a low power level. Within the fog layer, local area networks are installed, where the data are transmitted from endpoints to a gateway, where it is then transmitted to sources for processing and return transmission. By the end, data are transmitted to the cloud layer, which can access several IoT devices at the same time. It permits real-time and continuous data processing with higher computational capabilities. However, even though wearable devices are becoming more powerful and affordable, machine-learning-based tasks that typically require more computation resources may overload them with higher data communications and, therefore, higher energy consumption.

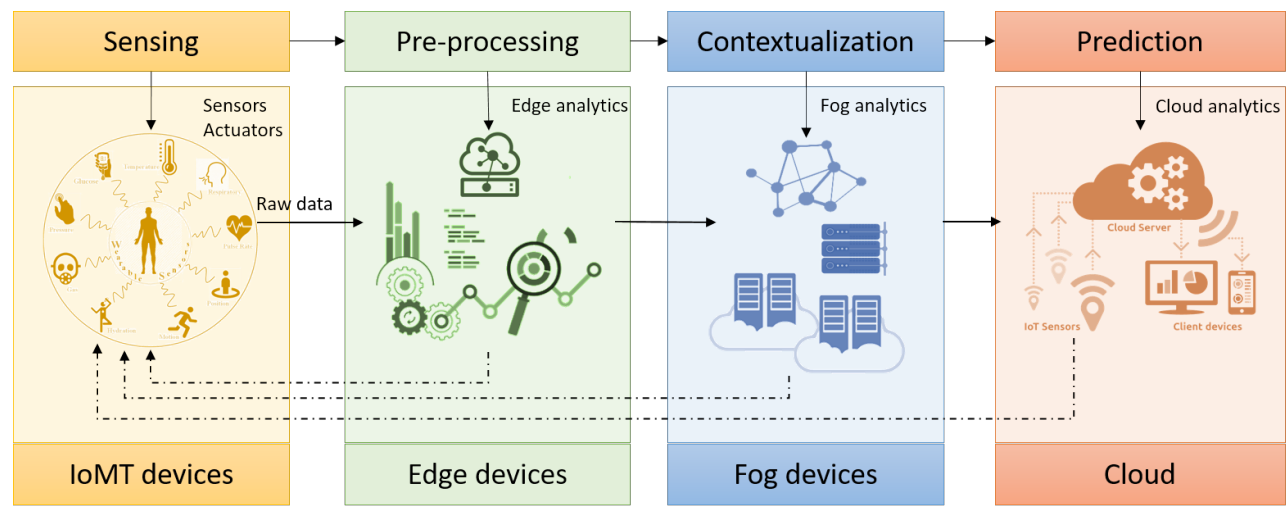

Figure 1. General layers architecture of IoMT system.

Therefore, it becomes imperative to offload some tasks from resource-constrained edge devices to co-located edge devices, such as the fog. Applications that require intensive computation resources are often offloaded to cloud servers to be processed, which improves IoT device capabilities. Cloud computing, by contrast, may cause high latency response times, privacy and security issues. As a solution, some studies proposed to offload tasks to a Mobile Edge Computing (MEC) server via edge devices that can be placed near end devices and process some computational capacity. Thus, transmission latencies are reduced, and reliability and security are enhanced. Even though computation offloading over fog edge computing or MEC has reduced the energy consumption of IoMT devices to a certain degree, their energy limitations remain a key concern. However, most devices are powered by batteries, which limits their energy resources and operating times. Similarly, 
computation performance may be affected if not enough battery energy is available for task transmission. A larger battery or more frequent recharging can address this problem. In contrast, the small size of IoMT devices makes it difficult to equip them with larger batteries or to recharge their batteries frequently. To address these challenges, energyharvesting technologies have been identified as promising techniques to increase battery life and achieve energy-autonomous systems. Figure 2 shows the general architecture of an IoMT system with the integration of EH-supplied systems and considering the taskoffloading aspect. The IoMT system includes various types of sensors used, most likely activity sensors (presented in red circles in Figure 2), physiological sensors (presented in green circles in Figure 2). Sensor are placed over the human body within a network, where each sensor is responsible for monitoring certain physical information. The sensor data are gathered in the base station to be transmitted to the next IoT layer, which can be either an access point, a gateway or a mobile device. Later, the collected data are transported to the fog layer and then the cloud. Communication can be established between different installed devices over the different layers. During the communication, information related to the actual status of the corresponding devices, such as the residual energy level, neighbor list and reception acknowledgement could be shared. This information care is used later to decide upon the most appropriate device for task offloading. Offloading involves sharing details about which device will be best suited to execute the current task, the type of task that will be executed, and how it will be executed. Task offloading can occur at different levels of the IoMT system, such as from the WBAN to the gateway and from the gateway to the fog, to the cloud.

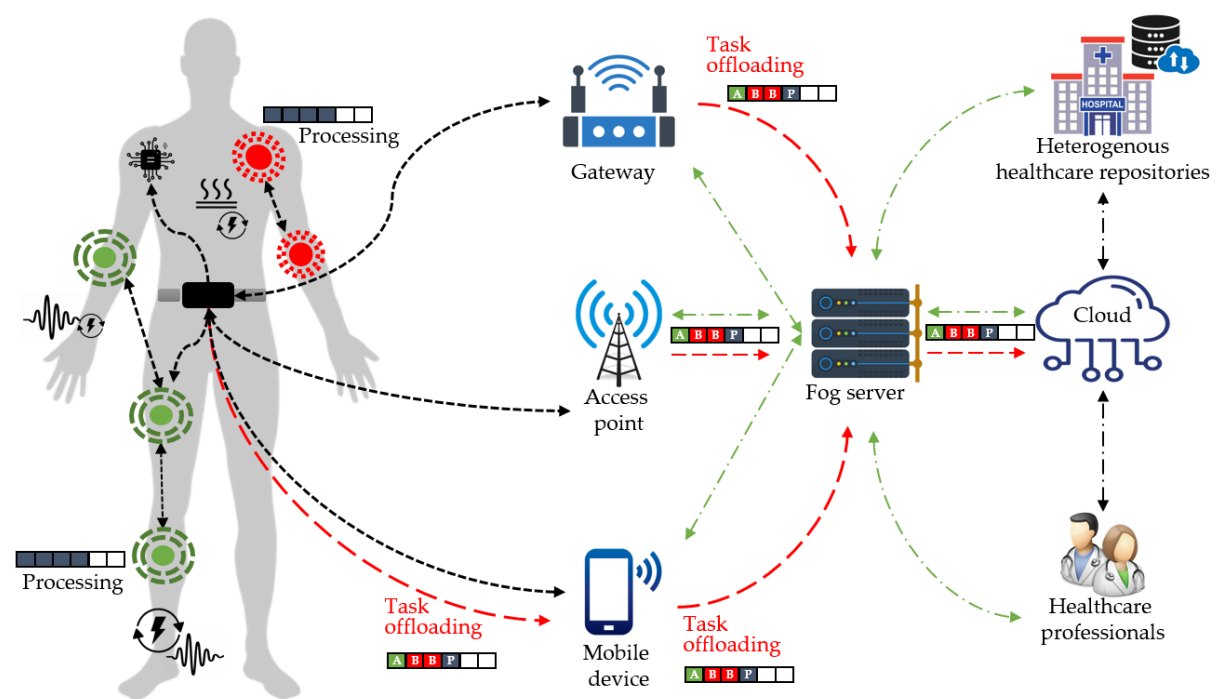

IoT Edge devices

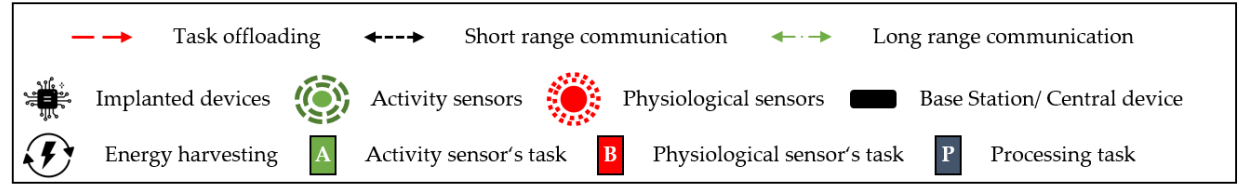

Figure 2. General architecture of an IoMT system based on energy harvesting and with consideration of task offloading.

Within the framework of IoT for medical applications, continuous data transmission takes place over the different layers of the network. Therefore, different sensor and communication technologies are used for sensing and transmitting data in real time, enabling fast calculations and optimal decision-making. It is crucial to satisfy the trade-off between the energy consumption, computational capability and data transmission for a real-time and accurate operation. Several schemes for energy efficiency and management are required to respond to these challenges. In general they can be classified into four main categories: 
- Resources allocation ensures a better allocation and management of the available resources, mainly radio and energy resources.

- Energy harvesting and transfer provide a sustainable energy supply, which are harvested from ambient resources. In the case of wearable systems, the energy can even be harvested from the human activities, such as breathing and movement.

- Hardware systems are explored during the development and design of wireless nodes and devices with consideration of the minimum energy consumption.

- Network installation enables the definition of appropriate infrastructures that maximize energy efficiency and ensure the data transmission and computational capabilities.

In this direction, it is important to investigate energy-efficient solutions for IoMT system, where intensive tasks and data processing are realized in a strict execution time. In particular, the communication and data transmissions need more attention, especially in the case of limited energy sources and computation capabilities. In this direction, investigations into energy-harvesting solutions along with task-offloading concepts present a promising solution to deal with excessive demands for a stable communication and data transmission. The contributions of this paper are:

- We provide a literature review of the state-of-the-art joint energy-harvesting and task-offloading approaches in fog edge computing systems.

- We compare the state-of-the-art related surveys based on specific key features.

- We investigate energy-harvesting technologies and energy-storage strategies for IoMT devices.

- We survey recent research efforts on task offloading in fog edge computing and related design considerations.

- We review existing approaches for the design of patient-centered care system.

The paper is organized as follows. Section 2 surveys research efforts related to joint energy harvesting and task-offloading approaches in fog edge computing systems. Section 3 presents the task-offloading approaches for fog edge computing, and deep-reinforcement learning-based algorithms. Section 4 highlights the related design considerations and challenges for EH driven task offloading. Section 5 reviews possibilities of energy supply, energy-storage strategies and recent trends in energy harvesting. Section 6 presents requirements for patient-centered care system. Finally, Section 7 concludes the paper.

\section{Related Works}

Recently, task offloading in fog edge computing systems has gained considerable attention due to the increasing development of IoMT devices. In [7], the authors developed a deep-learning-based, Internet of Medical Things-enabled edge computing framework for tackling COVID-19. It detects various COVID-19 symptoms and generates reports and alerts for medical decision support. Results indicate that the system can be used to effectively manage in-home health during a pandemic. Nevertheless, improvements to the system accuracy were needed as well as implementations with real subjects. In [8] a joint optimization framework was also proposed for IoT fog computing to achieve optimal resource allocation. The results show that the proposed framework enhanced the performance of IoT-based network systems. In [9] authors investigated delay-sensitive task offloading in edge-enabled healthcare services. A priority-aware service provisioning was proposed, allowing edge server computing resources to handle hard-deadline tasks earlier than soft-deadline tasks which have a lower priority and can tolerate longer delays over hard-deadline tasks. In contrast, the authors plan to examine how hard-deadline tasks can be placed in remote healthcare applications where ensuring high reliability is a crucial requirement.

When focusing on the increasing number of tasks that require high computational capability and consequently more energy, mobile devices need effective mechanisms to figure out which tasks to perform locally and which to migrate to the cloud. The authors in [10] discussed different computational offloading techniques. They consider the offloading either to a fog node or a cloud. They both have their trade-offs. The cloud, as an example, 
is rich in terms of resources, but offloading computational tasks to cloud servers can lead to security and privacy issues and it is also far away from mobile nodes. In contrast, fog is nearby but has limited resources. Hence, offloading to a cloud or fog consumes different amounts of energy and increases computation performance. In this context, the authors proposed an energy consumption-oriented algorithm to reduce energy consumption when offloading tasks. Initially, they compute the consumed energy when offloading the task to the fog compared to the cloud. Afterwards, they evaluated which entity would be preferred for the task based on the computation requirements. Based on these factors, the task is then offloaded to the desired entity.

Energy harvesting is a promising technology for converting ambient (solar, wind, etc.) and human energy (motion, breath) into electrical power, enabling communication systems to achieve energy-autonomous and efficient communications. In [11] the joint offloading and resource allocation issues in energy harvesting small cell networks is addressed to maximize the number of tasks performed by edge servers while reducing their energy and delay costs. In [12], the authors proposed a deep-reinforcement-learning-based framework for online offloading to reduce the computational complexity in large EH-driven networks. The proposed algorithm can successfully improve offloading behavior by implementing a deep neural network that learns binary offloading decisions based on past offloading experiences. In contrast, a distributed implementation of the proposed algorithm is still needed to enable the users to make offloading decisions in a distributed manner via a learning process. Similarly, a reinforcement-learning-based privacy-aware offloading scheme for a healthcare IoT device supplied by energy harvesting was proposed in [13]. The offloading policy applied on the edge device can be determined by considering the privacy level, energy consumption, and computation latency at each time slot. In [14], the authors investigated computation offloading and resource allocation issues with multiple energy harvesting supplied mobiles. All mobile devices initially harvest energy from RF signals and then use it to perform their own tasks locally or offload them to a MEC server. Some other offloading schemes can also achieve self-sustaining operations. In [15], for instance, the state-of-the-art of methodologies for task offloading in MEC and wireless power transfer to end nodes were recently described. The authors demonstrated the effective use of the Wireless Power Transfer (WPT) technique to charge end mobile phones which have gained more popularity in MEC. However, the increasing demand for computing resources may degrade the performance of MEC. Accordingly, they highlighted the influence of making decisions between task-offloading implementations and offloading locations on the power consumption of MEC devices.

Energy-efficient appliances have become prevalent in various fields and industries, including health care. Therefore, energy management is an effective technique for evaluating the energy efficiency of different devices. By contrast, the surveyed contributions lack discussions of joint energy-harvesting technologies, fog edge computing, and energy management techniques which are vital for IoMT devices.

Table 1 compares the state-of-the-art-related surveys based on specific key features.

Table 1. Comparison between state-of-the-art surveys.

\begin{tabular}{lllll}
\hline Reference & Fog Edge Computing & Task Offloading & Energy Harvesting & Energy Storage \\
\hline$[16-18]$ & $\checkmark$ & - & - & - \\
\hline$[19,20]$ & $\checkmark$ & $\checkmark$ & - & - \\
\hline This work & $\checkmark$ & $\checkmark$ & $\checkmark$ & $\checkmark$ \\
\hline
\end{tabular}

\section{Principles of Task Offloading}

\subsection{Pre-IoT Age}

Task or computation-offloading theory emerged to respond with the need to speed up task processing in hardware. Task migration in a distributed system aims at balancing 
the load among available processors without a drastic increase in the communication overhead [21,22]. Two classes of algorithms have been devised: static and dynamic. Communication protocol plays a pivotal role in balancing the load among processors. Three types of control models have been articulated for load balancing: centralized, distributed, and hybrid [23].

In a multicore/multiprocessor system, task offloading has been used to speed up the execution of multitasks, given a process $P_{1}$ that can be decomposed into $n$ independent processes, $P 1, n$ and $M$ cores. Each process $k$ requires an execution time $t_{k, m}$ on the mth core, such that $m \in\{1, \ldots, M\}$ and $k \in\{1, \ldots, n\}$ (see Appendix A). The energy dissipated by the $\mathrm{mth}$ core to run the kth process is $E_{k, m}$. The task offloading seeks an offloading algorithm that assigns tasks such that the execution time is met at the lowest possible energy consumption, i.e., the offloading should solve the following optimization problem.

$$
\begin{array}{ll}
\min & \sum_{m=1}^{M} \sum_{i=1}^{n} \delta_{i, m} E_{i, m} \\
\text { s.t. } & \sum_{k=1}^{n} t_{k, m} \leq t_{,}, \\
& n \leq M
\end{array}
$$

where $\delta_{i, m}\left\{\begin{array}{ll}1 & \text { if } P_{i} \text { runs on processor } m \\ 0 & \text { else }\end{array}\right.$.

The authors of [24] devised an offloading strategy that moves the computationally demanding task from CPU to GPU. They further demonstrated this strategy by considering the implementation of a signature-matching intrusion detection system. This approach has been generalized to cover the multicore architecture with and without accelerators.

Offloading can be used to balance the load among cores or processors in a multiprocessor system. This is often regarded as task migration that aims at moving the task execution from one core/processor using a given performance metric: power consumption, thermal energy, and dark silicon [25]. Communication-driven task migration attempts to migrate tasks to adjacent cores.

\subsection{Post IoT Age}

The Internet of Things, IoT, is the new trend in connectivity spawned from progress in sensors, embedded systems, and communication technologies. It is a three-tier architecture that is composed of a perception/sensors layer, connectivity layer, and application layer [26].

Mobile edge computing, MEC, is a new frontier in computing technologies. Multitude factors have contributed to the emergence of edge computing. Traditionally, cloud computing has been the dominant technology for the storage and processing of big data. Conventional task-offloading techniques have been proposed to migrate computationally intensive tasks / applications to cloud servers for processing. The offloading decision is aimed at either reducing end-user power consumption or increasing system performance [27]. However, the offloading strategies devised for cloud computing are not adequate in today's technologies for the following reasons: (1) Cloud servers cannot sustain the real-time processing of critical tasks, (2) the growing need for data protection and privacy, (3) the exponential increase in the number of IoT devices, and (4) the rising concern of the power consumption of data centers [28]. It has been reported that in the US, data centers consume up to $2.2 \%$ of all utility power [29]. According to the International Energy Agency (IEA), nearly $1 \%$ of global energy is consumed by data centers (roughly $250 \mathrm{TWh}$ ).

Edge computing, EC, addresses the shortcomings of cloud computing by bringing cloud-like services and operations close to the user. Task-offloading techniques have also been developed for edge computing. Fog computing is a term coined by CISCO and emerged after edge computing [30,31]. Fog-computing architecture, as illustrated in Figure 3, is composed of IoT end devices, fog devices that can perform processing and 
storage (such as micro cloudlet and gateways), and cloud layer (typically data centers and cloud servers) $[32,33]$.

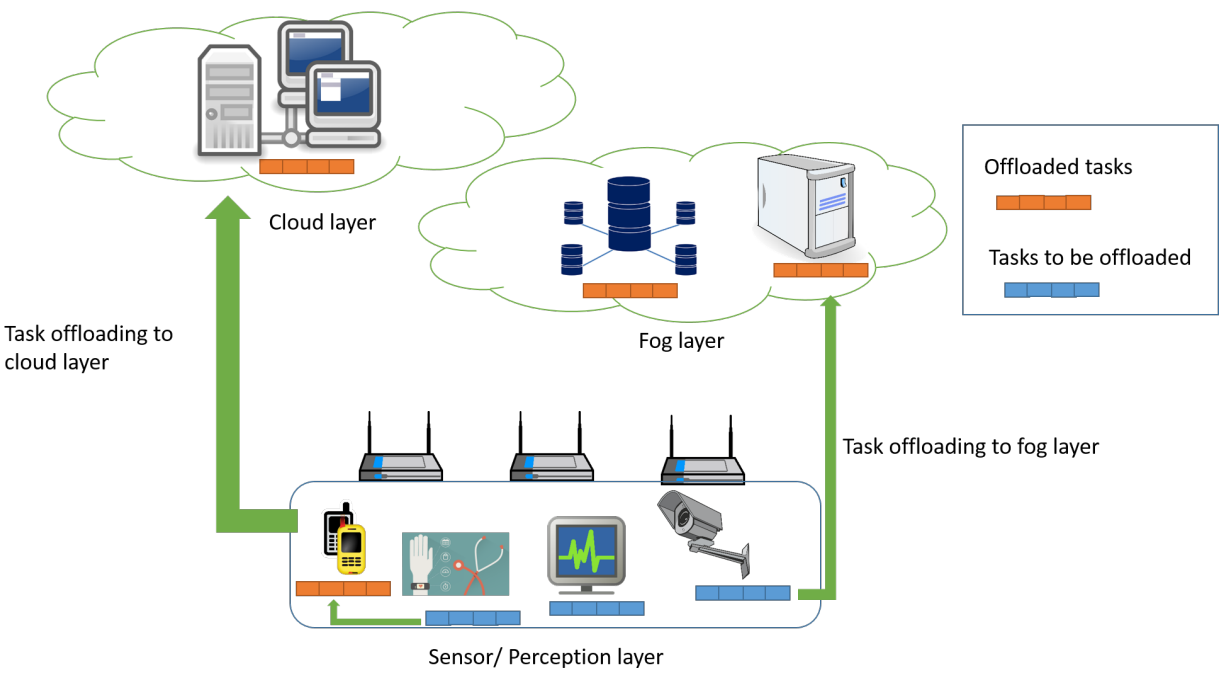

Figure 3. Offloading strategies using fog-computing paradigm. The fog layer is composed of cloudlets (small-scale data centers), and storage (fog servers). The cloud layer houses data centers and servers.

In the realm of the fog-computing paradigm, task offloading has become a hierarchical approach in which an offloading algorithm can execute the task locally using a specialized core, or nearby on an edge device, or remotely on fog or cloud nodes.

Task migration to near or far end nodes needs to account for the cost of the communication protocol: power and delay. In the context of fog computing, the offloading algorithm needs to solve the following optimization algorithm.

$$
\begin{array}{ll}
\min & \sum_{m=1}^{M} \sum_{i=1}^{n} \delta_{i, m}\left(E_{i, m}+E C_{i, m}\right) \\
\text { s.t. } & \sum_{k=1}^{n}\left(t_{k, m}+\tau_{k, m}\right) \leq t_{,} \\
& n \leq M
\end{array}
$$

where $E C_{i, m}$ is the energy consumed to transmit data of task $P_{i}$ to processor $m$, and $\tau_{k, m}$ is the latency to transmit task data to the processor $m$. Those parameters depend on the type of the communication protocol as well as the load of the remote processor that will execute the task.

\subsection{Offloading Algorithms}

The offloading algorithms aim to find a suitable processor (locally or remotely) to execute a task given a certain constraint. In wearables, offloading can be done at two stages: from wearables to edge devices or from edge device to fog/cloud devices [34]. The offloading device keeps on checking the estimated available power and compares it with the forested power demands. The offloading algorithms are invoked whenever the power demands exceed the available power (harvested and stored) and the energy consumed by the communication unit is less than the energy consumed by task processing. This concept is illustrated in Figure 4. Offloading can be combined with advanced techniques for power management such as sleep, dynamic voltage and frequency scaling (DVFS), and approximated computing [35]. 


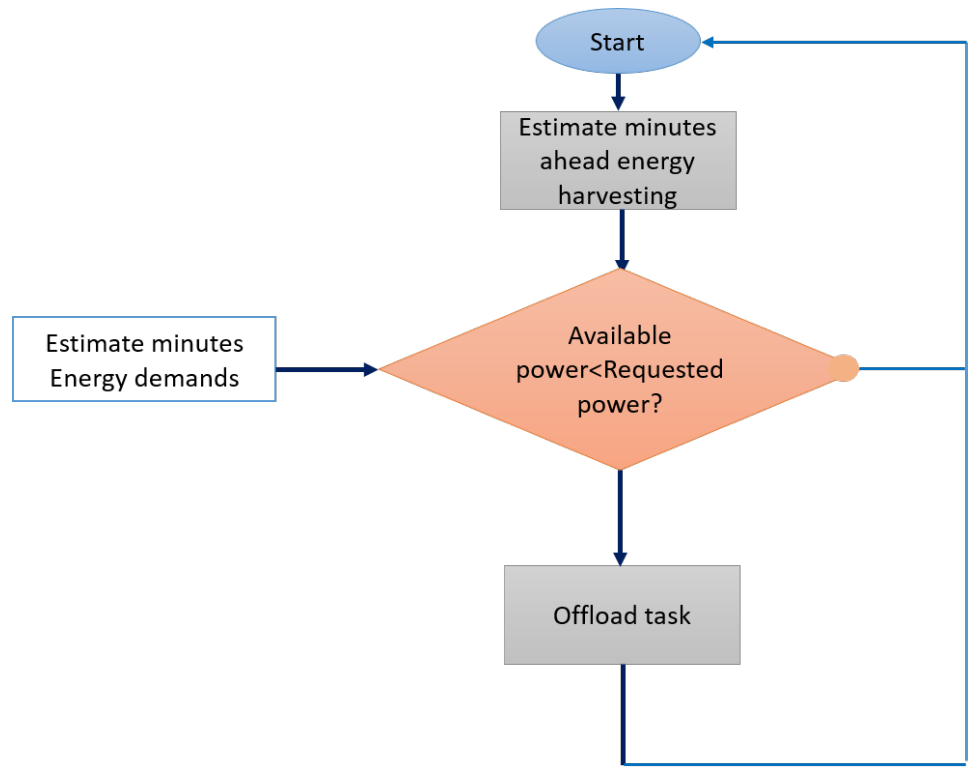

Figure 4. Principles of energy-aware offloading algorithm.

\subsubsection{Algorithm Classification}

Numerous offloading algorithms for fog computing have recently been proposed. Those algorithms belong to two categories: learning and non-learning. Table 2 summarizes the types of offloading algorithms proposed recently.

Table 2. Recently proposed offloading algorithm.

\begin{tabular}{cccc}
\hline Reference & Algorithm Type & Optimization Problem & Objective \\
\hline$[36]$ & Heuristic & Approximation algorithm & $\begin{array}{c}\text { Reduce energy consumption of } \\
\text { wearables }\end{array}$ \\
\hline$[37]$ & Heuristic & $\begin{array}{c}\text { Mixed-integer nonlinear } \\
\text { programming }\end{array}$ & $\begin{array}{c}\text { Joint scheduling and } \\
\text { offloading }\end{array}$ \\
\hline$[38]$ & $\begin{array}{c}\text { Coalition game } \\
\text { theory }\end{array}$ & merge and split & $\begin{array}{c}\text { maxi- mize the total numbers } \\
\text { of computed bits }\end{array}$ \\
\hline$[39]$ & Evolutionary & genetic algorithm & $\begin{array}{c}\text { joint optimization of load } \\
\text { balance and propagation delay }\end{array}$ \\
\hline$[40]$ & Deterministic & Iterative & balance relays energy \\
\hline$[41]$ & $\begin{array}{c}\text { Reinforcement } \\
\text { learning }\end{array}$ & $\begin{array}{c}\text { decentralized partially } \\
\text { observable Markov } \\
\text { decision process }\end{array}$ & $\begin{array}{c}\text { Maximizing IoT utility and } \\
\text { satisfying delay requirements }\end{array}$ \\
\hline$[43,44]$ & $\begin{array}{c}\text { Reinforcement } \\
\text { learning }\end{array}$ & $\begin{array}{c}\text { Deep Deterministic Policy } \\
\text { Gradient }\end{array}$ & $\begin{array}{c}\text { Maximizing task completion } \\
\text { rate and reducing task latency }\end{array}$ \\
\hline & learning & Q-deep learning & Reduce computation latency \\
\hline
\end{tabular}

\subsubsection{Deep-Reinforcement Learning}

In recent years, much attention has been given to deep-reinforcement learning (DRL) in task offloading. Reinforcement learning, RL, is a branch of artificial intelligence in which an agent interacts with the environment and learns using two functions: reward and punishment. Punishment is a negative reward. In RL, the learning cycle is not based on a training dataset; instead, the agent interacts with the environments with no prior knowledge and obtains immediate feedback based on its performance. The environment is modeled as a Markovian Decision Process (MDP). In RL an experience is defined as the triple $\left(s_{t}, a_{t}, r_{t}\right)$, where $s_{t}, a_{t}$, and $r_{t}$ are, respectively the state, action and reward at the time 
t. The agent determines the action based on a policy, $\pi(s)$. Q-learning algorithm is an offline policy that estimates $\pi(s)$ with guaranteed convergence. The mapping between the policy and the state at a given time $t$ is given by (3) [45]

$$
Q\left(s_{t}, a_{t}\right)=Q\left(s_{t}, a_{t}\right)+\alpha\left(r_{t}+\gamma \max _{a} Q\left(s_{t+1}, a_{t}\right)-Q\left(s_{t}, a_{t}\right)\right),
$$

where $\alpha$ is the learning rate, and $\gamma$ is the discount rate. In $R L$, the agent tends to maximize the rewards. This concept is illustrated in Figure 5.

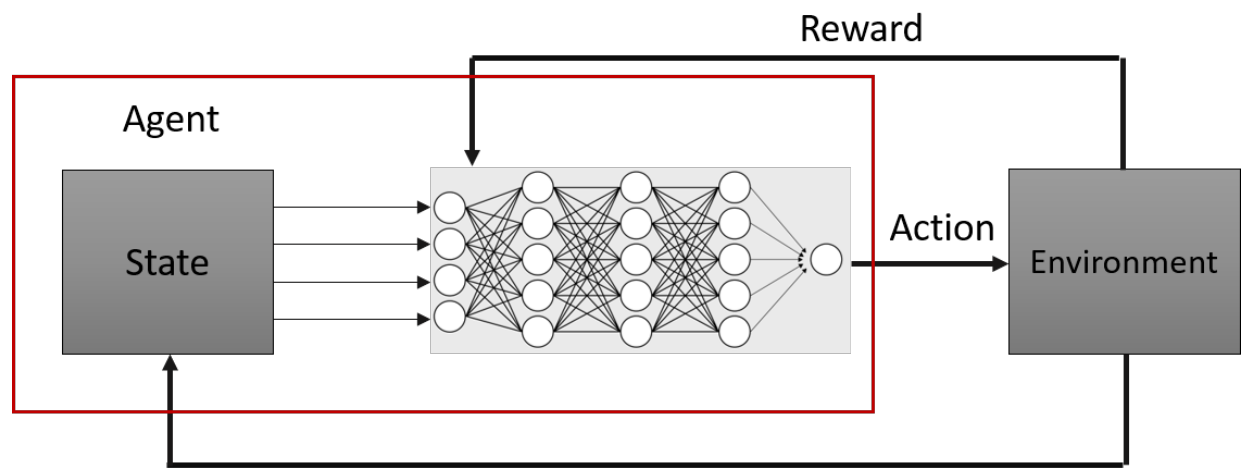

Figure 5. Principles of DRL.

Offloading algorithms-based Q-Learning has been devised in many published reports such $[11,43,44,46-48]$.

In [46], the authors devised a dynamic computation-offloading strategy for an MEC system using Markov decision process theory. The authors considered IoT devices with energy-harvesting techniques. The optimal offloading is achieved using a low-complex after-state learning method.

The problem of task offloading in the context of MEC has been formulated in [47] as a constrained Markov decision process (CMDP). The authors applied Lagrangian primaldual optimization and devised a deep-reinforcement learning algorithm to solve the relaxed CMDP.

Dynamic computational offloading for MEC systems with EH-enabled IoT devices considering multiple offloading servers has been studied and solved in [48]. The authors elaborated an offloading algorithm using deep Q-learning techniques.

Hardware implementation of the Q-learning algorithm received scant attention. Most of the reported implementation focuses on designing an accelerator using FPGA technology. The lowest power consumption has been reported to be $37 \mathrm{~mW}$ for a Q-matrix of dimensions eight states and four policies [49].

The work of [41] considered task offloading with energy harvesting for an IoT MEC system. The offloading problem has been formulated as a decentralized partially observable Markov decision process. They further reduced the computational complexity by searching for an approximated solution using an RL decentralized offloading algorithm. The results, obtained using Matlab simulations, showed that the proposed reduces both average delay and average energy consumption.

To this end, it is crucial to identify the power consumption during different activities of the end devices, in particular data processing, data transmission and communication. In the following section, specific design considerations for task offloading are presented, which may influence the power consumption and the latency of the software and hardware components of the IoMT systems. The main focus is attributed to the choice of the communication protocols for IoT devices with consideration of the energy consumption.

\section{Design Considerations for the Task Offloading}

With respect to the general architecture of IoMT, Wireless Body Area Networks (WBANs) $[38,50,51]$ are installed where various types of sensors are used, most likely 
activity sensors (e.g., accelerometer), physiological sensors (e.g., heart rate, ECG and body temperature) and environmental sensors (e.g., humidity and air pressure) (see Figure 1). Various types of applications are recognized with enhanced sensing and communication capability, such as biomedical and wearable solutions for health monitoring, human activities control, organ implantation monitoring and remote surgical interventions. These applications require a high data rate, low latency and high quality of services (QoS) [39,40,52], in order to ensure precise, real-time and secure medical applications. With the integration of the IoMT, it is more challenging to identify the most appropriate strategy that enables efficient handling of the intensive and continuous requests from the installed wireless devices on the human body promptly. Moreover, wireless sensor nodes are battery powered, where the lifetime of the battery is directly dependent of the number of executed tasks along the process. Due to this, it is important to increase the computation capacity of battery-powered devices when performing intensive computing tasks while ensuring real-time intervention and data transmission. In this context, the choice of suitable data transmission and communication protocols has a strong influence on the evaluation of the task-offloading algorithm in terms of processing time, energy consumption and computation. In the following, an overview of the most common communication protocols is provided.

\subsection{Communication Protocols}

The general architecture of IoMT is reported in Figure 1. The provided architecture is composed of three main layers: (1) the things/devices layer, where the WBAN is installed, along with the gateway devices, (2) the fog layer, and (3) the cloud layer [53,54]. Different communication technologies can be identified within each layer, which enable transmission of the data from the end devices to the end user.

In the first layer, different sensor nodes are installed on the body, which build the WBAN. Sensor nodes can be implantable, wearable or mobile, placed for example in the hand of the patient. In this type of network the communication between sensors is carried out within a short range of 2 to $3 \mathrm{~m}$. These devices, basically, require small power sources with respect to the safety and security of the user. Therefore, with respect to the low power specification and small communication range, the standard Industrial Scientific and Medical (ISM) band is sufficient to cover the installed nodes [55,56]. Various communication technologies are supported in the ISM band, such as ZigBee, Bluetooth and Wi-Fi. Moreover, alternative communication technology is introduced such as the Intra-Body Communication (IBC) technology [57,58]. Through IBC, the human body is used as a transmission medium, enabling power-saving, and thus improving the robustness and security of communications. Due to these advantages, IBC has been included as a third physical layer in the IEEE 802.15.6 standard for wireless body area networks designated as Human Body Communication (HBC) [59]. A central device, refereed also as base station, is responsible for collecting sensor data and forwarding them to the next communication layer. Accordingly, intermediate devices are installed as a bridge between the small interconnected WBAN and the exterior local network, namely the Wireless Local Area Network (WLAN). In this case, local gateways are used such as mobile devices, access points or simple midlayer gateways. Typically, they provide a bridge between the IoT edge devices and the fog and cloud servers. They enable the passing of data from the discrete sensor network to the other cloud and application layers. On one side, common communication technologies can be initiated between the WBAN' nodes and the intermediate gateways, such as the Wi-Fi and Bluetooth. In the other side, communication with the fog and cloud server can be realized through $5 \mathrm{G}, \mathrm{Wi}-\mathrm{Fi}$ or GPR $[60,61]$. To this end, data communication and storage are carried out over this layer, whereas in the IoT layer, installed wireless devices are periodically transmitting information. Sensor devices remain awake for a specific time frame from time to time to transmit the required information.

Within the second layer, local servers and gateway devices for the fog network are placed. These devices enable the processing of the collected data. Excessive and complex 
processing and data-mining algorithms can be carried out at this stage. Later, the collected data are redirected to the cloud layer for further processing. In the case of the cloud or fog layer, more powerful and long-range protocols are required, namely the LoRaWAN, Sigfox, NB-IoT and LTW-M [62], which ensure a better coverage range with a minimum of $1 \mathrm{~km}$ in urban deployment and $10 \mathrm{~km}$ in rural deployment. Moreover, the fog layer is in connection with healthcare experts responsible, which permits a reduction to the time delay of the interpretation and execution of specific tasks and decisions. In the third layer, powerful data storage and computation resources are installed. In this instance data analysis, decision-making and urgent intervention can be recognized. In addition, the cloud layer permits the incorporation of various and heterogeneous healthcare systems, which enables a real-time and continuous access to the current patient, equipment and planned tasks supervision and monitoring. Basically, this layer consists of cloud-based resources that will store the data generated by the medical infrastructure and be used to perform analytical work as needed in the future [54]. An overview of the common communication technologies used in WBANs is presented in Table $3[63,64]$.

Table 3. Comparison between communication technologies used in the WBANs.

\begin{tabular}{|c|c|c|c|c|c|}
\hline Criteria & Range in $\mathrm{m}$ & Data Rate & Frequency & Standard & Energy Consumption \\
\hline Bluetooth $[65,66]$ & $<10$ & $1-3$ Mbits/s & $2.4 \mathrm{GHz}$ & IEEE 802.15.1- & $<30 \mathrm{~mA}$ \\
\hline NFC [67] & 0.1 & $424 \mathrm{Kbit} / \mathrm{s}$ & $13.56 \mathrm{MHz}$ & ISO/IEC 1800-3 & $<15 \mathrm{~mA}$ \\
\hline RFID $[68,69]$ & $<12$ & $100 \mathrm{Mbit} / \mathrm{s}$ & $\begin{array}{l}\text { LF: } 125-135 \mathrm{KHz}, \\
\mathrm{HF}: 13.56 \mathrm{MHz}, \\
\text { UHF: } 868-930 \mathrm{MHz}, \\
\text { Microwave } 2.45,5.8 \mathrm{GHz},\end{array}$ & ISO/IEC 1800 & - \\
\hline BLE [66] & $10-300$ & $125 \mathrm{Mbit} / \mathrm{s}$ & $2.4 \mathrm{GHz}$ & IEE 802.15 .1 & $<15 \mathrm{~mA}, 10-100 \mathrm{~mW}$ \\
\hline ZigBee $[66,70]$ & $10-500$ & $250 \mathrm{Kbit} / \mathrm{s}$ & $2.4 \mathrm{GHz}$ & IEEE 802.15 .4 & $<16 \mathrm{~mA}, 10-100 \mathrm{~mW}$ \\
\hline Wi-Fi $[64,70]$ & 100 & $11 \mathrm{Mbit} / \mathrm{s}$ & $2.4,5 \mathrm{GHz}$ & IEEE $802.11 \mathrm{a} / \mathrm{b} / \mathrm{g}$ & - \\
\hline LoRaWAN [71,72] & $\begin{array}{l}\sim 5 \text { in urban } \\
20 \text { in rural }\end{array}$ & $\begin{array}{l}56 \text { bits/s UL } \\
296 \text { bits/s DL }\end{array}$ & $868,434,915 \mathrm{MHz}$ & LPWAN & $\begin{array}{l}\text { Sleep: } 7.66 \mu \mathrm{A} \text { to } 34 \mathrm{~mA} \\
\text { Tx: } 133 \mathrm{~mA} \\
\text { Rx: } 16.3 \mathrm{~mA}\end{array}$ \\
\hline Sigfox $[73,74]$ & $\begin{array}{l}\sim 10 \mathrm{~km} \text { in urban } \\
\sim 40 \mathrm{~km} \text { in rural }\end{array}$ & $\begin{array}{l}100 \text { bits/s UL } \\
60 \text { bits/s DL }\end{array}$ & $868,434,915 \mathrm{MHz}$ & LPWAN, UNB & $\begin{array}{l}\text { Sleep: } \sim 1 \mu \mathrm{A}, \mathrm{Tx}: 49 \mathrm{~mA} \\
\text { Rx: } 19 \mathrm{~mA}\end{array}$ \\
\hline NB-IoT [75-77] & $\begin{array}{l}\sim 1 \mathrm{~km} \text { in urban } \\
\sim 10 \mathrm{~km} \text { in rural }\end{array}$ & $220 \mathrm{Kbits} / \mathrm{s}$ & Licensed LTE & LPWAN & $\begin{array}{l}\text { Sleep: } 13 \mathrm{~mW} \\
\text { Tx: } 716 \mathrm{~mW}, \mathrm{Rx}: 21 \mathrm{~mW}\end{array}$ \\
\hline
\end{tabular}

\subsection{Energy Consumption of Wireless Nodes}

Typically, in the task-offloading paradigm, computing tasks are created by end devices (e.g., wireless sensor nodes, central devices). Therefore, the energy requirement at the level of the wireless node, as well as the network, are emphasized. Therefore, characterizing the energy consumption of the end device is crucial to create a balance between the energy requirement, demands and consumption. Essentially, the wireless sensor node is composed of four main units: energy management unit, communication unit, data processing unit and sensing unit. The energy management unit is responsible for converting the energy retrieved from either the battery or the energy-harvesting circuit into a suitable energy level, which can be used to supply the electronics of the node. Using energy harvesting helps to reduce the dependency on the battery power by extending the lifetime of the node itself [78]. The communication unit contains the radio transceiver module used for wireless communication. The processing unit is the core of the node, where all data processing and node activity is carried out. The last unit contains the embedded sensors, which can be either passive or active and are responsible for the sensing and actuating tasks. Basically, the effective lifetime of the node is dependent on the available, residual energy and the required amount of energy to successfully carry out the assigned task. Consequently, the total energy consumption is deducted in relation to the energy supply and energy consumption during data processing and communication. Considering the 
energy provided by the harvesting module and the module consumption, the effective residual energy at a time instance $t$ is estimated in accordance with the consumed, harvested and residual energy amounts of the previous time instance.

$$
E_{\text {Res }}(t)=E_{\text {Res }}(t-1)-E_{\text {Cons }}+E_{\text {Harv }}
$$

$E_{\text {Res }}(t), E_{\text {Res }}(t-1), E_{\text {Cons }}$ and $E_{\text {Harv }}$ are the residual energy of the node at a time instance $t$, the residual energy at a time instance $(t-1)$, energy consumption and the energy of the harvesting module, respectively (see Appendix A).

The general definition of the energy consumption within a sensor node is presented in Equation (5).

$$
E_{\text {Cons }}=E_{\text {Transceiver }}+E_{\text {System }}+E_{\text {Sensing }}
$$

where $E_{\text {Transceiver }}, E_{\text {System }}$ and $E_{\text {Sensing }}$ are the energy consumed during the reception and transmission of data packet, energy consumed within the coding and decoding activities and the energy consumed during sensing activities, respectively.

With respect to the standard energy consumption model, the $E_{\text {Transceiver }}$ is presented based on the transmitter and receiver electronic definition as presented in Equations (7) and (9). The total energy consumption, within the radio module during data transmission, becomes:

$$
E_{\text {Cons }}=E_{R_{x}}+E_{T_{x}}+E_{\text {System }}+E_{\text {Sensing }}
$$

The energy of transmission and reception are dependent on the number of transmitted data bits over a distance $d$, where $E_{\text {elec }}$ is the electrical energy of the circuitry needed to transmit or to receive a $l$ bit data packet. $d$ is the distance between the receiver and transmitter.

$$
E_{T_{x}}=\left\{\begin{array}{cc}
E_{\text {elec }} \times l+E_{f s} \times l \times d^{2}, & d \leq d_{T} \\
E_{\text {elec }} \times l+E_{\text {amp }} \times l \times d^{4}, & d>d_{T}
\end{array}\right.
$$

The distance between both transmitter and receiver is dependent on the medium access and therefore, it is defined based on $\epsilon_{f}$ and $\epsilon_{a m p}$, which present the energy consumption factor for free space and for the multipath radio models, respectively. The threshold distance $d_{T}$ is defined as:

$$
d_{T}=\sqrt{\frac{\epsilon_{f s}}{\epsilon_{a m p}}}
$$

The energy consumption during the reception is defined based on the number of communicated bits $l$, which is defined in Equation (9). The list of the used parameters with their typical values is illustrated in Appendix A.

$$
E_{R_{x}}(l)=E_{\text {elec }} \times l
$$

Eventually, the effective energy consumption of a wireless node depends strongly on how often it sends and receives data packets, and processes sensor information.

Task offloading offers a promising solution to reduce the workload on the installed devices, by adopting specific algorithms where the task realization is offloaded to devices with efficient energy sources and computation capabilities. In the context of WBANs and wearable solutions, intensive computing is mitigated from the wireless sensor to the edge and from the cloud to the fog. It presents an efficient solution to manage the intensive communication and computation in a limited energy source environment. Moreover, by adopting an energy-harvesting solution, the energy of the system can be kept available to carry out the assigned tasks in real time and continuously, which remains challenging for different applications, such as in the case of real-time and continuous pulse monitoring [79], motion tracking [80], exoskeleton manipulation [81] and the maintenance and monitoring of implantable devices [82]. To this end, providing continuous and efficient power supply to wearable and implantable devices presents a highly addressed challenge in recent 
research [83-85]. As part of this, integrating energy-harvesting technologies with taskoffloading approaches allows end devices to endure for a long time to support long-term task processing [86-88].

\section{Power Supply for Wearables with Task Offloading Capabilities}

Task-offloading approaches can be efficiently combined with energy harvesting to address the issue of insufficient battery capacity and limited computation resources in IoMT devices and consequently increase the operating time of wearable devices. This is referred to as joint energy harvesting and task offloading. Using this technology, users can extract energy, convert it into useful energy, store it in the appropriate energy-storage device, and use that energy to perform the corresponding local computing and offloading tasks [15,89-91].

As depicted in Figure 4, the offloading algorithm reacts based on two estimations: The energy harvested/stored and the energy demands. Energy harvesting from ambient sources is considered a promising solution that can be used to provide power supply for IoMT devices and thus replace batteries. The most commonly used harvesters for the supply of wearable devices are piezoelectric harvesters, thermoelectric generators, RF harvesters, and solar cells. Table 4 illustrates the amount of power that can be harnessed from different sources, along with some advantages and limitations associated with each.

Ambient light presents the highest power density among other sources, with the possibility of harvesting indoor and outdoor. However, it has limited application due to its restricted availability.

Table 4. Available power from different energy sources (literature survey).

\begin{tabular}{|c|c|c|c|}
\hline Energy Source & Harvested Power & Advantages & Disadvantages \\
\hline \multicolumn{4}{|l|}{ Mechanical energy } \\
\hline Human (motion) & $4 \mu \mathrm{W} / \mathrm{cm}^{2}$ & \multirow{2}{*}{$\begin{array}{l}\text { High power } \\
\text { density }\end{array}$} & \multirow{2}{*}{$\begin{array}{l}\text { Depending on the } \\
\text { source properties }\end{array}$} \\
\hline Industry (vibrations) & $100 \mu \mathrm{W} / \mathrm{cm}^{2}$ & & \\
\hline \multicolumn{4}{|l|}{ Thermal energy } \\
\hline Human (heat) & $25 \mu \mathrm{W} / \mathrm{cm}^{2}$ & \multirow{2}{*}{$\begin{array}{c}\text { Widely } \\
\text { available }\end{array}$} & \multirow{2}{*}{$\begin{array}{l}\text { Limitation of } \\
\text { power density }\end{array}$} \\
\hline Industry & $1-10 \mathrm{~mW} / \mathrm{cm}^{2}$ & & \\
\hline \multicolumn{4}{|l|}{ Ambient light } \\
\hline Indoor & $10 \mu \mathrm{W} / \mathrm{cm}^{2}$ & \multirow{2}{*}{$\begin{array}{l}\text { High power } \\
\text { density }\end{array}$} & \multirow{2}{*}{ Intermittent } \\
\hline Outdoor & $10 \mathrm{~mW} / \mathrm{cm}^{2}$ & & \\
\hline \multicolumn{4}{|l|}{ Radio frequency } \\
\hline GSM & $0.1 \mu \mathrm{W} / \mathrm{cm}^{2}$ & \multirow{2}{*}{$\begin{array}{l}\text { Widely } \\
\text { available }\end{array}$} & $\begin{array}{l}\text { Power dependent on } \\
\text { distance between }\end{array}$ \\
\hline Wi-Fi & $0.001 \mathrm{~mW} / \mathrm{cm}^{2}$ & & RF source-harvester \\
\hline
\end{tabular}

\subsection{Thermoelectric Generators}

Energy can be derived from heat using thermoelectric generators (TEGs) based on the thermoelectric effect. It is also known as the Seebeck effect, according to which electricity is generated by the temperature gradient between two conductors. A TEG can be attached to the body to convert the temperature difference between a body skin and the surrounding environment into voltage. This concept was launched in 1999, where the first wristwatch supplied by body heat was invented [92]. TEGs can be used as an efficient power supply for wearable devices when the human body and the surrounding environment have a temperature difference of 5 to 10 degrees.

The electric potential of a TEG is expressed by Equation (10)

$$
V_{T E G}=S \cdot \Delta T
$$


where $S$ is the Seebeck coefficient of the material used and $(\Delta T)$ is the temperature difference across the TEG.

A thermally powered wearable device that incorporates an accelerometer to sense falls was developed in [93]. In this application, the device generated $520 \mu \mathrm{W}$ of output power at $15{ }^{\circ} \mathrm{C}$, which charged a capacitor and a power management unit, included to link the thermal source and a sensor node.

An in-depth analysis of thermoelectric generation technology was recently presented in [94], illustrating the working principles of TEGs and their applications. Nevertheless, the development of thermoelectric materials with acceptable power factors remains a major challenge, for which various techniques have been investigated to achieve better efficiencies.

\subsection{Kinetic Energy Harveters}

In contrast to solar or thermal energy, a kinetic energy source is not dependent on location or time. Kinetic harvesters are based on the extraction of vibration or motion and the conversion of the mechanical energy into electrical power through one or a combination of different transduction mechanisms. The most common ones are piezoelectric, electromagnetic, electrostatic, and triboelectric. These harvesters are classified related to their transduction mechanisms. Unlike other means of transduction, piezoelectric harvesters directly convert human motion changes into electrical signals without any requirements for further external input. Piezoelectric (PE) harvesters operate through the piezoelectric effect. When a force is applied to a PE element, a mechanical strain is developed, causing the material to exhibit changes in its polarization, causing the accumulation of electrical charges across the piezoelectric material. The changes in charge distribution produce an electric field depending on the applied force, frequency of oscillation, and geometry of the harvester.

Electromagnetic kinetic energy harvesters operate based on Faraday's law induction which states that once a conductor moves through an electric field, a current is induced. A system of springs, magnets and coils are used in electromagnet energy-harvesting systems. Coil number and magnetic mass are the main determinants of the output power of these energy harvesters. Therefore, reducing their size, weight and complexity is challenging. As example, the authors of [95] demonstrated the effectiveness of a frequencyconverted electromagnetic harvester which extracts energy mainly from human limb motion. A power density of $0.33 \mathrm{~mW} / \mathrm{cm}^{3}$ was achieved in this work using low-frequency human vibration to power wearable devices at extremely low frequencies.

\subsection{Flexible Piezoelectric Generators}

The body is an excellent source of significant amounts of mechanical energy which can be produced from several biological processes, including walking, heartbeat, breathing and muscle movements. Thanks to their high flexibility, piezoelectric nanogenerators (PENGs) can convert this mechanical stress into electrical charges through nanostructured piezoelectric materials when stretched, pressed or flexed. In addition, this technology can potentially be integrated with other energy-harvesting mechanisms, resulting in hybrid energy-harvesting solutions. The simple architecture of PENGs makes them attractive and considered to be the most promising energy harvesters for wearable devices and microsystems. The materials used in piezoelectricity are diverse, including crystals, ceramics, and polymers. The converter needs to be attached to a part of the body subjected to strong compressive stress to maximize the piezoelectric effect. PENGs can provide enough power to supply devices with power consumption ranging from microwatts to milliwatts, which best fits the wearable sensor range as seen in Table 5 where the energy consumption of typical wearable sensors is presented. 
Table 5. Energy consumption of typical medical sensors.

\begin{tabular}{lcll}
\hline Wearable Sensors & $\begin{array}{c}\text { Voltage } \\
\text { Range }\end{array}$ & $\begin{array}{c}\text { Power } \\
\text { Consumption }\end{array}$ & Description \\
\hline $\begin{array}{l}\text { Optical heart rate sensors } \\
\text { - BH1790GLC optical heart } \\
\text { rate sensor [96] }\end{array}$ & $1.7-3.6 \mathrm{~V}$ & $720 \mu \mathrm{W}$ & $\begin{array}{l}\text { Measures the pulse waves } \\
\text { that occur when the heart } \\
\text { pumps blood. }\end{array}$ \\
\hline $\begin{array}{l}\text { Blood pressure sensors } \\
\text { - Capacitive tactile sensor [97] }\end{array}$ & $1.8-3.3 \mathrm{~V}$ & $1.2-4.6 \mathrm{~mW}$ & $\begin{array}{l}\text { Measures the pressure } \\
\text { exerted by the circulating } \\
\text { blood on the walls of blood } \\
\text { vessels. }\end{array}$ \\
$\begin{array}{l}\text { Glucometers } \\
\text { - Implantable RFID glucose } \\
\text { monitoring sensor [98] }\end{array}$ & $1.0 \mathrm{~V}-1.2 \mathrm{~V}$ & $50 \mu \mathrm{W}$ & $\begin{array}{l}\text { Measures the average } \\
\text { blood glucose concentration. }\end{array}$ \\
\hline $\begin{array}{l}\text { Pulse oximeter sensors } \\
\text {-MAX30102 pulse oximetry [99] }\end{array}$ & $1.8 \mathrm{~V}-3.3 \mathrm{~V}$ & $<1 \mathrm{~mW}$ & $\begin{array}{l}\text { It attaches to a body part, } \\
\text { most commonly to a finger } \\
\text { to measure the oxygen } \\
\text { saturation level of the } \\
\text { circulating arterial blood. }\end{array}$ \\
\hline
\end{tabular}

Flexible Piezoelectric generators can be modeled as sinusoidal current sources $I_{P}$ in parallel with parasitic capacitances $C_{P}$ and internal resistances $R_{P}$ when excited by sinusoidal vibrations at their resonant frequencies. Since the piezoelectric transducer can deliver an alternating irregular $\mathrm{AC}$ current rather than direct current (DC), an electronic interface is essential to enable voltage compatibility between the piezoelectric element and the load. The electronic interface greatly influences the energy-harvesting effectiveness [100], which has driven a variety of research efforts to develop PENG-compatible energy management interfaces [101]. Implementing these circuits is mostly intended to allow the user to use irregular AC power harnessed by piezoelectric transducers (PTs) to supply loads such as wearable sensors. The rectification stage of PEH systems is usually coupled with a DC-DC converter [100] to scale the rectified voltage to match the application's requirements.

One limitation of the classic AC-DC energy-harvesting circuits when implemented with PEts is that negative output power is produced because the output current and voltage could not keep the same phase, leading to a loss of an amount of the harvested energy. P-SSHI and S-SSHI have been proposed to overcome this limitation. The main difference between the circuits is how we connect the switch $S$ and the inductor $L$, either in series, so we are talking about SSHI or in parallel to deal with P-SSHI. When the vibration occurs, the switch $\mathrm{S}$ remains open, allowing the current to flow through the circuit to the storage element $\mathrm{Cr}$. If the piezoelectric element's voltage drops below a certain threshold, the switch S will automatically close, inverting the voltage across the PE element and therefore stopping current flow. This means that the switch is kept closed until a full inversion of the PEt's voltage has been achieved. Nevertheless, this voltage inversion causes an electrical damping that opposes the mechanical vibrations on the piezoelectric material. This effect is known as Synchronized Switch Damping (SSD). It can significantly affect the overall conversion efficiency, and it is consequently the main limitation of both PSSHI and S-SSHI circuits. Figures 6 and 7 display the P-SSHI and S-SSHI energy-harvesting interfaces, respectively. 


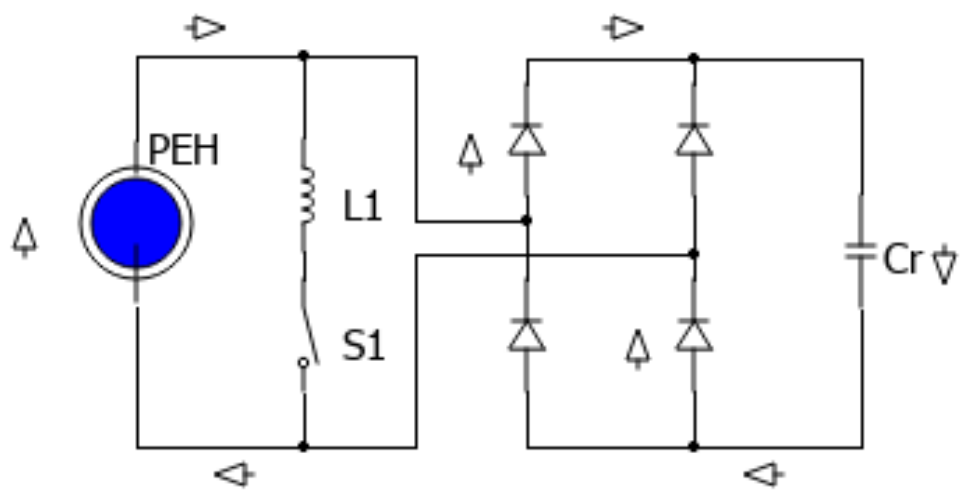

Figure 6. Schematic of P-SSHI energy extraction interface.

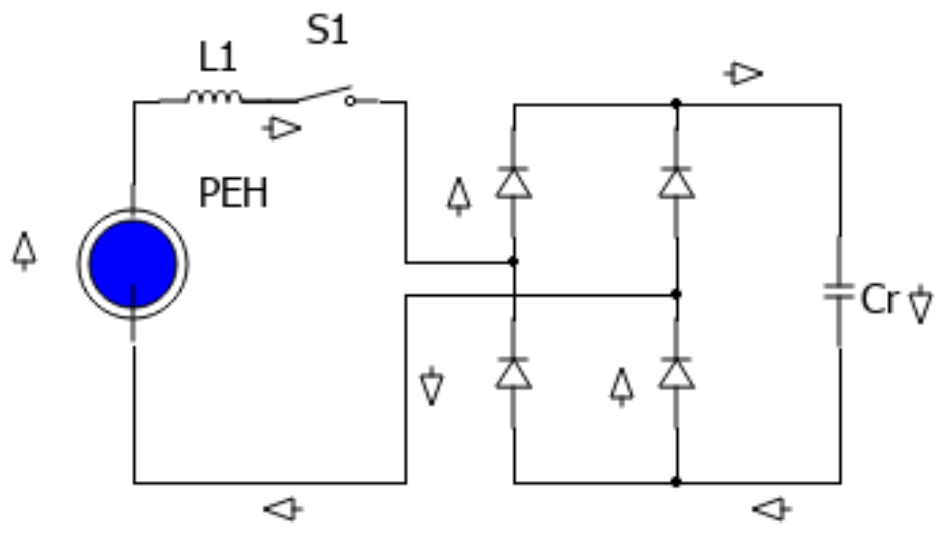

Figure 7. Schematic of S-SSHI energy extraction interface.

SECE circuit, displayed in Figure 8, mainly prevents the SSD effect, which is the main limitation of P-SSHI and S-SSHI circuits. This effect is caused by the direct connection between the output load and the piezoelectric transducer during the hole vibration phase. When the PEH generates the voltage, the switch $S$ will be closed, and the energy will be stored in the inductor $\mathrm{L}$ as seen in the figure.

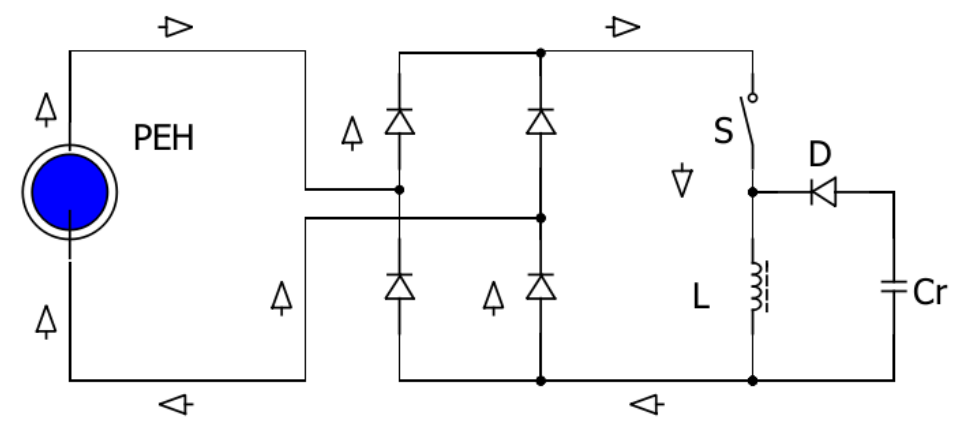

Figure 8. Schematic of SECE energy extraction interface when the switch S is closed.

When the vibration stops, the voltage across the piezoelectric element falls to zero, and the switch $S$ will open immediately. Consequently, the energy accumulated in the inductor will be directly transferred to the storage capacitor and the load. One limitation for this interface is the complexity when compared to the simple architecture and switching strategy that characterize SSHI circuits.

The control of the integrated switches was a common limitation for the reviewed interfaces, so several researchers were focusing on developing self-powered resonant energy-harvesting circuits. In [102], authors demonstrated an optimized self-powered P- 
SSHI circuit that can automatically switch once the voltage exceeds its maximum threshold. In addition, this technology can potentially be integrated with other energy-harvesting mechanisms, resulting in hybrid harvesting solutions [103].

\subsection{Energy-Storage Techniques}

Using energy harvesting to achieve battery-free operation has gained high interest. However, any interruption in the energy-harvesting source will affect the wearable device's operation. Therefore, an energy-storage mechanism is still required to maintain a smooth power supply for charge loads and serve as a backup whenever the energy source is unavailable.

The harnessed energy can be stored before being supplied to the MCU, or the power can be delivered directly. The decision of whether implementing a storage element in a wearable device considers different factors:

- $\quad$ Placement of the device: implant or outside.

- $\quad$ Energy source: type

- $\quad$ Requirements of the application: either it needs a sustainable supply for the wearable device or a non-critical usage.

Batteries and super capacitors are the two main solutions for energy storage. Energy storage for wearable devices must comply with several requirements. First, the storage element needs to be rechargeable to avoid frequent battery replacements, which can be inconvenient in several cases. In pacemakers, as an example, surgery needs to be performed every eight years to replace their lithium batteries [104]. As a second requirement, the storage device needs to be capable of supporting long-term application with minimal impact on battery parameters.

The following Table 6 compares two storage mechanisms, batteries and capacitors.

Table 6. Comparison between different storage techniques for energy harvesters in IoMT devices

\begin{tabular}{lll}
\hline Comparison & Conventional Batteries & Supercapacitors \\
\hline Storage mechanism & Chemical & Physical \\
Energy storage & High & Limited \\
Recharging cycles & $100 \mathrm{~s}$ & Millions \\
Charging time & Hours & Sec-minutes \\
Impedance & Low-high & Low \\
Physical size & Large & Medium \\
Capacity & $0.3-2500 \mathrm{mAH}$ & $10-100 \mu \mathrm{AH}$ \\
\hline
\end{tabular}

They differ mostly in the number of charging cycles since capacitors can reach millions of cycles. In addition, capacitors require only a few seconds for charging, so the charging time is very fast compared to batteries. In contrast, supercapacitors cannot be used in AC and high frequency circuits and have lower capacity than batteries, but this can satisfy the requirements of some low-power applications. One more limitation for using batteries as a storage element is that the battery is susceptible to leakage, leading to chemical poisoning, especially when used in implants. Batteries can leak chemicals when overcharged or heated (above $60^{\circ} \mathrm{C}$ ). This can lead to chemical burns risking human beings. Due to their advantages over batteries, super capacitors are a promising alternative to store energy. In a super capacitor, thin dielectric layers and electrodes hold power at the electrode-electrolyte interface to be accessed when needed. Thanks to their high pulse power capacity, they can also handle small power surges. Super capacitors' excellent cycle lifetime also makes them ideally suited to act as energy-storage components in energy-harvesting-based sustainable power systems.

\subsection{Recent Energy-Harvesting Solutions for Wearables}

The human body can be a versatile source of energy harvesting $[105,106]$. Energy can be harvested from everyday activities, such as breathing, arm motion, walking, running, 
or pedaling, without performing a specific workout. The body can produce mechanical energy through various body zones movements, such as the elbow, the knee, the ankle or the heels. The performance of three vibrating generators was studied in [107] at nine different body locations for a person walking on a treadmill. The results indicate that the energy generated at lower-body locations (hip, knee, and ankle) is four times greater than the energy generated at upper-body locations. Additionally, body heat offers promising possibilities for supplying wearable systems. Based on the Seeback effect, a flexible TEG generated $4.95 \mathrm{~mW}$ of body heat and was used for a wearable multi-sensing bracelet [108]. A energy-autonomous, multi-sensing bracelet can operate under varying conditions, including human motion. The amount of energy in such systems is highly dependent on the temperature difference between the human body and the ambient environment [109]. Several studies have shown that physiological activities, such as blood pressure, heart motion and breathing, can regularly provide wearable devices with energy supply. In [110], cardiac contractions are used to supply low-powered pacemakers. When powered by a constant heartbeat of $90 \mathrm{bpm}$, the harvester can deliver $11.1 \mathrm{j}$ of electrical energy. Because of the small size and weight requirement, energy extraction from the human body is much more complicated than energy harvesting from machines [111]. The available power is often weak and difficult to use, such as human kinetic energy, which typically has a low frequency and a low amplitude.

Recently, thermoelectric nanogenerators (TENGs) were demonstrated as a conventional technique for rehabilitation in [112]. As an exercise gaming device, a wearable TENG-based rehabilitation device (Rehab-TENG) was developed. The device was used to control a game on a laptop by flexing and extending the arm. It is an effective way of testing the motor function of an impaired arm. Rehab-TENG is also used as an energy harvester in an exercise system where the patient moves an impaired arm to store energy in a capacitor. It is possible to assess the level of deficiency by measuring the charging rate of the storage element, which consequently enhances patients' motivation for exercising more repetitive movements of the impaired body zone. This, in turn, speeds up recovery. Furthermore, the authors suggested using the Rehab-TENG device as an autonomous home exercise and monitoring system, which is particularly relevant during pandemics, therefore reducing the necessity for hospital visits for rehabilitation.

An emerging trend in energy-harvesting technologies for IoMT is developing biocompatible wearable harvesters, such as textiles, footwear, or watches, which are energyautonomous, lightweight, flexible, and have more computational resources for better performance. Consequently, various energy-saving approaches were proposed to mitigate the problem of excessive energy demands during the operation of devices. Task offloading is a promising and effective technique that extends the operating time of wearables by migrating the energy intensive task to edge device. Task-offloading algorithms attempts to solve an optimization problem by looking for a suitable remote processor to perform the offloading, taking into consideration the overheads caused by the communication link (energy and latency). Real-time implementation of task offloading for wearables is still in its infancy.

\section{Value-Based Healthcare System and Personalized Healthcare}

The legacy health care system is staff-centric. Driven by the need to transform the healthcare system to be patient- and personnel-centric, numerous governments have proposed a transformation strategy. For instance, in Saudi Arabia, the government has identified eight challenges that the current health care system should cope with. Those challenges are: (1) the continued growth and aging of the population, (2) the prevalence of avoidable injuries and non-communicable diseases beyond the international standard, (3) the inadequacy and inconsistency of primary care, (4) wide-scale disparity in the quality of care, (5) a significant deficiency in value and quality, (6) the system is resource-, staff-, and institution-centric, (7) insufficient use of digital integrated systems, and (8) the growing need to decrease government spending in health care systems [113]. 
The value-based healthcare system is a new framework adopted by many governments to improve healthcare services and user experience through the improvement of patient healthcare outcomes at the lowest possible cost, i.e., the value is determined as the ratio of outcomes to cost [114]. Preventive medicine and early intervention lowers the cost associated with the hospitalization of patients. Healthcare 4.0, a new paradigm shift in the health industry, has transformed healthcare from an institution-centered to a patientcentered system [115].

Wearables are cornerstone technologies in Healthcare 4.0. The design of patientcentered care mandates the inclusion of the user requirements to identify functional and non-functional requirements [35]. Surveys, focus groups, and interviews are common ways to capture user requirements. In [116], the authors devised a cost-efficient system for the monitoring of the sedentary level of senior citizens. The system requirements and guidelines have been gathered from a literature review. The system is then evaluated using a mixed approach: focus group, interview, and observations. The system is refined through the feedback provided by the end-user. The authors reported that: (1) the majority of the respondents are interested in receiving a feedback on the level of their physical activity at the end of the day, (2) nearly $58 \%$ of participants showed interest in a system that integrates games with physical activity, and (3) virtually $83 \%$ of the participants showed interest in profiling their daily activities and receiving alerts when their physical activities are low.

The user requirements for the wearables targeting Chinese seniors are the focus of the work described in [117]. Those requirements have been classified under the following three categories: healthcare requirements, privacy and security requirements, and commodity requirements.

\section{Conclusions}

Wearable devices are the heart of IoMT. Energy-harvesting techniques can achieve energy-autonomous wearable devices. However, handling tasks that require intensive computing resources limits their performance. To overcome these limitations, energy-aware task-offloading approaches were proposed to reduce the device energy consumption and improve computation resources. This paper surveys recent works on joint task offloading and energy-harvesting techniques in the IoMT. In addition, possibilities of power supply for medical sensors and energy-storage strategies are investigated.

Joint task offloading and energy harvesting is still an active area of research. The offloading is meaningful at two possible levels: from wearables (IoT end device) to edge devices (IoT high-end or middle-end device), or from edge devices to fog nodes. An off-policy-based reinforcement learning algorithm has been often proposed in the literature. Nevertheless, its hardware implementation has received scant attention.

Future work will focus on the efficient hardware implementation of joint energy harvesting and reinforcement learning-based task offloading for wearable devices. Nevertheless, privacy and security might affect the offloading strategy when applied to wearables; this topic was not considered in this study.

Author Contributions: M.B.A., I.B.D. and D.E.H. contributed equally to the manuscript concept, methodology, original draft writing, visualization and editing. S.S., A.F. and O.K. contributed by reviewing, visualization and editing. I.B.D. secured the funding of the manuscript. All authors have read and agreed to the published version of the manuscript.

Funding: The publication of this article was funded by Chemnitz University of Technology.

Institutional Review Board Statement: Not applicable.

Informed Consent Statement: Not applicable.

Conflicts of Interest: The authors declare no conflict of interest. 


\section{Abbreviations}

$\mathrm{EH}$

SSHI

P-SSHI

S-SSHI

SECE

SEH

SSD

EWMA

HBC

IoT

IoMT

MCU

MEC

QoS

WBANs

WSNs

PENGs

PEH

FPEGs

PEt

TEG
Energy Harvesting

Synchronized Switch Harvesting on Inductor

Parallel Synchronized Switch Harvesting on Inductor

Serial Synchronized Switch Harvesting on Inductor

Synchronized Electrical Charge Extraction

Standard Energy Harvesting (Bridge rectifier)

Synchronized Switch Damping

Exponentially Weighted Moving Average

Human Body Communication

Internet of Things

Internet of Medical Things

Micro-Controller Unit

Mobile Edge Computing

Quality of Service

Wireless Body Area Networks

Wireless Sensor Networks

Piezoelectric nanogenerators

Piezoelectric energy harvesting

Flexible Piezoelectric Generators

Piezoelectric transducer

Thermoelectric Generator

\section{Appendix A}

Table A1. List of parameters with their typical values.

\begin{tabular}{|c|c|c|c|}
\hline Parameter & Explanation & Typical Value/Range & Unit \\
\hline$E_{\text {Res }}(t)$ & Residual energy at a time instance $t$ & NA & $\mathrm{J}$ \\
\hline$E_{\text {Res }}(t-1)$ & $\begin{array}{l}\text { Residual energy at a time instance } \\
\qquad(t-1)\end{array}$ & NA & $\mathrm{J}$ \\
\hline$E_{\text {Cons }}$ & Energy consumption & NA & $\mathrm{J}$ \\
\hline$E_{\text {Harv }}$ & Energy of the harvesting module & NA & $\mathrm{J}$ \\
\hline$E_{\text {Transceiver }}$ & Consumed energy of transmission & NA & $\mathrm{J}$ \\
\hline$E_{\text {System }}$ & $\begin{array}{l}\text { Consumed energy of coding and } \\
\text { decoding }\end{array}$ & NA & $\mathrm{J}$ \\
\hline$E_{\text {Sensing }}$ & Consumed energy of the sensing & NA & $\mathrm{J}$ \\
\hline$E_{R_{x}}$ & Consumed energy of the reception & NA & $\mathrm{J}$ \\
\hline$E_{T_{x}}$ & $\begin{array}{l}\text { Consumed energy of the } \\
\text { transmission }\end{array}$ & NA & $\mathrm{J}$ \\
\hline$E_{\text {elec }}$ & Electrical energy of the circuitry & Based on initial assumption (e.g., 50 nJ) & $\mathrm{J}$ \\
\hline$d$ & $\begin{array}{c}\text { Distance between transmitter and } \\
\text { receiver }\end{array}$ & Related to the realized scenario & $\mathrm{m}$ \\
\hline$d_{T}$ & $\begin{array}{l}\text { Threshold distance between } \\
\text { transmitter and receiver }\end{array}$ & $1 \mathrm{~m}$ & $\mathrm{~m}$ \\
\hline$l$ & Size of the data packet & Depends on the ADC of the processor & bits \\
\hline$\epsilon_{f}$ & $\begin{array}{l}\text { Energy consumption factor for free } \\
\text { space }\end{array}$ & Depends on the & $\mathrm{pJ} / \mathrm{bit} / \mathrm{m}^{2}$ \\
\hline$\epsilon_{a m p}$ & $\begin{array}{c}\text { Energy consumption factor for } \\
\text { multipath radio models }\end{array}$ & propagation loss & $\mathrm{pJ} / \mathrm{bit} / \mathrm{m}^{4}$ \\
\hline$M$ & Number of cores & 8 & - \\
\hline$n$ & Number of tasks & maximum value is 6 & - \\
\hline$\alpha$ & the learning rate & $10^{-4}$ & - \\
\hline$\gamma$ & the discount rate & 0.85 & - \\
\hline
\end{tabular}




\section{References}

1. Farias, F.A.C.d.; Dagostini, C.M.; Bicca, Y.d.A.; Falavigna, V.F.; Falavigna, A. Remote patient monitoring: A systematic review. Telemed. E-Health 2020, 26, 576-583. [CrossRef] [PubMed]

2. Tian, S.; Yang, W.; Grange, J.M.L.; Wang, P.; Huang, W.; Ye, Z. Smart healthcare: Making medical care more intelligent. Glob. Health J. 2019, 3, 62-65. [CrossRef]

3. Gatouillat, A.; Badr, Y.; Massot, B.; Sejdić, E. Internet of Medical Things: A Review of Recent Contributions Dealing With Cyber-Physical Systems in Medicine. IEEE Internet Things J. 2018, 5, 3810-3822. [CrossRef]

4. Yang, W.; Wang, S.; Sahri, N.M.; Karie, N.M.; Ahmed, M.; Valli, C. Biometrics for Internet-of-Things Security: A Review. Sensors 2021, 21, 6163. [CrossRef]

5. Swaroop, K.N.; Chandu, K.; Gorrepotu, R.; Deb, S. A health monitoring system for vital signs using IoT. Internet Things 2019, 5, 116-129. [CrossRef]

6. Ali, M.; Ali, A.A.; Taha, A.E.; Dhaou, I.B.; Gia, T.N. Intelligent Autonomous Elderly Patient Home Monitoring System. In Proceedings of the ICC 2019-2019 IEEE International Conference on Communications (ICC), Shanghai, China, 20-24 May 2019; pp. 1-6. [CrossRef]

7. Rahman, M.A.; Hossain, M.S. An Internet of medical things-enabled edge computing framework for tackling COVID-19. IEEE Internet Things J. 2021, 8, 15847-15854. [CrossRef]

8. Zhang, H.; Xiao, Y.; Bu, S.; Niyato, D.; Yu, F.R.; Han, Z. Computing resource allocation in three-tier IoT fog networks: A joint optimization approach combining Stackelberg game and matching. IEEE Internet Things J. 2017, 4, 1204-1215. [CrossRef]

9. Mukherjee, M.; Kumar, V.; Maity, D.; Matam, R.; Mavromoustakis, C.X.; Zhang, Q.; Mastorakis, G. Delay-sensitive and Priorityaware Task Offloading for Edge Computing-assisted Healthcare Services. In Proceedings of the GLOBECOM 2020-2020 IEEE Global Communications Conference, Taipei, Taiwan, 7-11 December 2020; pp. 1-5. [CrossRef]

10. Zhao, X.; Zhao, L.; Liang, K. An energy consumption oriented offloading algorithm for fog computing. In International Conference on Heterogeneous Networking for Quality, Reliability, Security and Robustness; Springer: Berlin/Heidelberg, Germany, 2016; pp. 293-301.

11. Zhang, Z.; Yu, F.R.; Fu, F.; Yan, Q.; Wang, Z. Joint offloading and resource allocation in mobile edge computing systems: An actor-critic approach. In Proceedings of the 2018 IEEE Global Communications Conference (GLOBECOM), Abu Dhabi, United Arab Emirates, 9-13 December 2018; IEEE: Piscataway, NJ, USA, 2018; pp. 1-6.

12. Huang, L.; Feng, X.; Zhang, C.; Qian, L.; Wu, Y. Deep reinforcement learning-based joint task offloading and bandwidth allocation for multi-user mobile edge computing. Digit. Commun. Netw. 2019, 5, 10-17. [CrossRef]

13. Min, M.; Wan, X.; Xiao, L.; Chen, Y.; Xia, M.; Wu, D.; Dai, H. Learning-based privacy-aware offloading for healthcare IoT with energy harvesting. IEEE Internet Things J. 2018, 6, 4307-4316. [CrossRef]

14. Wang, F.; Zhang, X. Dynamic computation offloading and resource allocation over mobile edge computing networks with energy harvesting capability. In Proceedings of the 2018 IEEE International Conference on Communications (ICC), Kansas City, MO, USA, 20-24 May 2018; IEEE: Piscataway, NJ, USA, 2018; pp. 1-6.

15. Mustafa, E.; Shuja, J.; Jehangiri, A.I.; Din, S.; Rehman, F.; Mustafa, S.; Maqsood, T.; Khan, A.N. Joint wireless power transfer and task offloading in mobile edge computing: A survey. Clust. Comput. 2021, 1-20. [CrossRef]

16. Engineer, M.; Tusha, R.; Shah, A.; Adhvaryu, D.K. Insight into the Importance of Fog Computing in Internet of Medical Things (IoMT). In Proceedings of the 2019 International Conference on Recent Advances in Energy-Efficient Computing and Communication (ICRAECC), Nagercoil, India, 7-8 March 2019; pp. 1-7. [CrossRef]

17. Hartmann, M.; Hashmi, U.S.; Imran, A. Edge computing in smart health care systems: Review, challenges, and research directions. Trans. Emerg. Telecommun. Technol. 2019, e3710. [CrossRef]

18. Omoniwa, B.; Hussain, R.; Javed, M.A.; Bouk, S.H.; Malik, S.A. Fog/Edge Computing-Based IoT (FECIoT): Architecture, Applications, and Research Issues. IEEE Internet Things J. 2019, 6, 4118-4149. [CrossRef]

19. Aazam, M.; Zeadally, S.; Flushing, E.F. Task offloading in edge computing for machine learning-based smart healthcare. Comput. Netw. 2021, 191, 108019. [CrossRef]

20. Islam, A.; Debnath, A.; Ghose, M.; Chakraborty, S. A survey on task offloading in Multi-access Edge Computing. J. Syst. Archit. 2021, 118, 102225. [CrossRef]

21. Ryou, J.C.; Wong, J. A task migration algorithm for load balancing in a distributed system. In Proceedings of the Twenty-Second Annual Hawaii International Conference on System Sciences. Volume II: Software Track, Kailua-Kona, HI, USA, 3-6 January 1989; Volume 2, pp. 1041-1048. [CrossRef]

22. Suen, T.; Wong, J. Efficient task migration algorithm for distributed systems. IEEE Trans. Parallel Distrib. Syst. 1992, 3, 488-499. [CrossRef]

23. Jiang, Y. A Survey of Task Allocation and Load Balancing in Distributed Systems. IEEE Trans. Parallel Distrib. Syst. 2016, 27, 585-599. [CrossRef]

24. Jacob, N.; Brodley, C. Offloading IDS Computation to the GPU. In Proceedings of the 2006 22nd Annual Computer Security Applications Conference (ACSAC'06), Miami Beach, FL, USA, 11-15 December 2006; pp. 371-380. [CrossRef]

25. Liu, C.; Tang, F.; Hu, Y.; Li, K.; Tang, Z.; Li, K. Distributed Task Migration Optimization in MEC by Extending Multi-Agent Deep Reinforcement Learning Approach. IEEE Trans. Parallel Distrib. Syst. 2021, 32, 1603-1614. [CrossRef] 
26. Sun, L.; Jiang, X.; Ren, H.; Guo, Y. Edge-Cloud Computing and Artificial Intelligence in Internet of Medical Things: Architecture, Technology and Application. IEEE Access 2020, 8, 101079-101092. [CrossRef]

27. Kumar, K.; Liu, J.; Lu, Y.H.; Bhargava, B. A survey of computation offloading for mobile systems. Mob. Netw. Appl. 2013, 18, 129-140. [CrossRef]

28. Jalali, F.; Hinton, K.; Ayre, R.; Alpcan, T.; Tucker, R.S. Fog Computing May Help to Save Energy in Cloud Computing. IEEE J. Sel. Areas Commun. 2016, 34, 1728-1739. [CrossRef]

29. Zhang, X.; Lu, J.J.; Qin, X.; Zhao, X.N. A high-level energy consumption model for heterogeneous data centers. Simul. Model. Pract. Theory 2013, 39, 41-55. [CrossRef]

30. Naha, R.K.; Garg, S.; Georgakopoulos, D.; Jayaraman, P.P.; Gao, L.; Xiang, Y.; Ranjan, R. Fog Computing: Survey of Trends, Architectures, Requirements, and Research Directions. IEEE Access 2018, 6, 47980-48009. [CrossRef]

31. Aazam, M.; Zeadally, S.; Harras, K.A. Offloading in fog computing for IoT: Review, enabling technologies, and research opportunities. Future Gener. Comput. Syst. 2018, 87, 278-289. [CrossRef]

32. Ud Din, I.; Guizani, M.; Hassan, S.; Kim, B.S.; Khurram Khan, M.; Atiquzzaman, M.; Ahmed, S.H. The Internet of Things: A Review of Enabled Technologies and Future Challenges. IEEE Access 2019, 7, 7606-7640. [CrossRef]

33. Kumari, A.; Tanwar, S.; Tyagi, S.; Kumar, N. Fog computing for Healthcare 4.0 environment: Opportunities and challenges Comput. Electr. Eng. 2018, 72, 1-13. [CrossRef]

34. Yang, Y.; Geng, Y.; Qiu, L.; Hu, W.; Cao, G. Context-Aware Task Offloading for Wearable Devices. In Proceedings of the 2017 26th International Conference on Computer Communication and Networks (ICCCN), Vancouver, BC, Canada, 31 July-3 August 2017; pp. 1-9. [CrossRef]

35. Ben Dhaou, I.; Ebrahimi, M.; Ben Ammar, M.; Bouattour, G.; Kanoun, O. Edge Devices for Internet of Medical Things: Technologies, Techniques, and Implementation. Electronics 2021, 10, 2104. [CrossRef]

36. Kalantarian, H.; Sideris, C.; Mortazavi, B.; Alshurafa, N.; Sarrafzadeh, M. Dynamic Computation Offloading for Low-Power Wearable Health Monitoring Systems. IEEE Trans. Biomed. Eng. 2017, 64, 621-628. [CrossRef]

37. Geng, Y.; Yang, Y.; Cao, G. Energy-Efficient Computation Offloading for Multicore-Based Mobile Devices. In Proceedings of the IEEE INFOCOM 2018-IEEE Conference on Computer Communications, Honolulu, HI, USA, 16-19 April 2018; pp. 46-54. [CrossRef]

38. Wang, L.; Shao, H.; Li, J.; Wen, X.; Lu, Z. Optimal Multi-User Computation Offloading Strategy for Wireless Powered Sensor Networks. IEEE Access 2020, 8, 35150-35160. [CrossRef]

39. Jiang, J.; Zhang, X.; Li, S. A Task Offloading Method with Edge for 5G-Envisioned Cyber-Physical-Social Systems. Secur. Commun. Netw. 2020, 2020, 8867094. [CrossRef]

40. Liao, Y.; Yu, Q.; Han, Y.; Leeson, M. Relay-Enabled Task Offloading Management for Wireless Body Area Networks. Appl. Sci. 2018, 8, 1409. [CrossRef]

41. Tang, Q.; Xie, R.; Yu, F.R.; Huang, T.; Liu, Y. Decentralized computation offloading in IoT fog computing system with energy harvesting: A dec-POMDP approach. IEEE Internet Things J. 2020, 7, 4898-4911. [CrossRef]

42. Li, Y.; Qi, F.; Wang, Z.; Yu, X.; Shao, S. Distributed Edge Computing Offloading Algorithm Based on Deep Reinforcement Learning. IEEE Access 2020, 8, 85204-85215. [CrossRef]

43. Gao, Z.; Hao, W.; Han, Z.; Yang, S. Q-Learning-Based Task Offloading and Resources Optimization for a Collaborative Computing System. IEEE Access 2020, 8, 149011-149024. [CrossRef]

44. Zhang, K.; Zhu, Y.; Leng, S.; He, Y.; Maharjan, S.; Zhang, Y. Deep Learning Empowered Task Offloading for Mobile Edge Computing in Urban Informatics. IEEE Internet Things J. 2019, 6, 7635-7647. [CrossRef]

45. Sutton, R.S.; Bach, F.; Barto, A.G. Reinforcement Learning, 2nd ed.; MIT Press Ltd.: Cambridge, MA, USA, 2018.

46. Wei, Z.; Zhao, B.; Su, J.; Lu, X. Dynamic Edge Computation Offloading for Internet of Things With Energy Harvesting: A Learning Method. IEEE Internet Things J. 2019, 6, 4436-4447. [CrossRef]

47. Zhang, K.; Samaan, N. Optimized Look-Ahead Offloading Decisions Using Deep Reinforcement Learning for Battery Constrained Mobile IoT Devices. In Proceedings of the 2020 IEEE International Conference on Smart Cloud (SmartCloud), Washington, DC, USA, 6-8 November 2020; pp. 181-186. [CrossRef]

48. Zhang, J.; Du, J.; Wang, J.; Shen, Y. Hybrid Decision Based Deep Reinforcement Learning For Energy Harvesting Enabled Mobile Edge Computing. In Proceedings of the 2020 International Wireless Communications and Mobile Computing (IWCMC), Limassol, Cyprus, 15-19 June 2020; pp. 2100-2105. [CrossRef]

49. Spanò, S.; Cardarilli, G.C.; Di Nunzio, L.; Fazzolari, R.; Giardino, D.; Matta, M.; Nannarelli, A.; Re, M. An Efficient Hardware Implementation of Reinforcement Learning: The Q-Learning Algorithm. IEEE Access 2019, 7, 186340-186351. [CrossRef]

50. Rawat, P.; Singh, K.D.; Chaouchi, H.; Bonnin, J.M. Wireless sensor networks: A survey on recent developments and potential synergies. J. Supercomput. 2013, 68, 1-48. [CrossRef]

51. Jung, J.; Lee, W.; Kim, H. Cooperative Computing System for Heavy-Computation and Low-Latency Processing in Wireless Sensor Networks. Sensors 2018, 18, 1686. [CrossRef]

52. Zhou, G.D.; Yi, T.H. Recent Developments on Wireless Sensor Networks Technology for Bridge Health Monitoring. Math. Probl. Eng. 2013, 2013, 947867. [CrossRef]

53. Razdan, S.; Sharma, S. Internet of Medical Things (IoMT): Overview, Emerging Technologies, and Case Studies. IETE Tech. Rev. 2021, 1-14. [CrossRef] 
54. Vishnu, S.; Ramson, S.J.; Jegan, R. Internet of Medical Things (IoMT)—An overview. In Proceedings of the 2020 th International Conference on Devices, Circuits and Systems (ICDCS), Coimbatore, India, 5-6 March 2020; IEEE: Piscataway, NJ, USA, 2020. [CrossRef]

55. Alabidi, E.S.; Kamarudin, M.; Rahman, T.; Khalily, M.; Abdulrahman, A.; Jamlos, M.; Jais, M. Radiation characteristics improvement of monopole antenna for WBAN applications. Int. J. Multimed. Ubiquitous Eng 2014, 9, 53-64. [CrossRef]

56. Al Islam, N.; Arifin, F. Performance analysis of a miniaturized implantable PIFA antenna for WBAN at ISM band. In Proceedings of the 2016 3rd International Conference on Electrical Engineering and Information Communication Technology (ICEEICT), Dhaka, Bangladesh, 22-24 September 2016; IEEE: Piscataway, NJ, USA, 2016; pp. 1-5.

57. Asan, N.B.; Hassan, E.; Perez, M.D.; Joseph, L.; Berggren, M.; Voigt, T.; Augustine, R. Fat-Intrabody Communication at 5.8 GHz Including Impacts of Dynamics Body Movements. 2019. Available online: http://www.diva-portal.org/smash/record.jsf?pid= diva2:1353288 (accessed on 10 January 2022).

58. Čuljak, I.; Lučev Vasić, Ž.; Mihaldinec, H.; Džapo, H. Wireless Body Sensor Communication Systems Based on UWB and IBC Technologies: State-of-the-Art and Open Challenges. Sensors 2020, 20, 3587. [CrossRef] [PubMed]

59. Álvarez-Botero, G.A.; Hernández-Gómez, Y.K.; Telléz, C.E.; Coronel, J.F. Human body communication: Channel characterization issues. IEEE Instrum. Meas. Mag. 2019, 22, 48-53. [CrossRef]

60. Windha M.V.; Iskandar; Hendrawan; Arifianto, M.S. Wireless Sensor Network on 5G Network. In Proceedings of the 2018 4th International Conference on Wireless and Telematics (ICWT), Bali, Indonesia, 12-13 July 2018; pp. 1-5. [CrossRef]

61. Papadopoulos, G.Z.; Kritsis, K.; Gallais, A.; Chatzimisios, P.; Noel, T. Performance evaluation methods in ad hoc and wireless sensor networks: A literature study. IEEE Commun. Mag. 2016, 54, 122-128. [CrossRef]

62. Aldahdouh, K.A.; Darabkh, K.A.; Al-Sit, W. A survey of 5G emerging wireless technologies featuring LoRaWAN, Sigfox, NB-IoT and LTE-M. In Proceedings of the 2019 International Conference on Wireless Communications Signal Processing and Networking (WiSPNET), Chennai, India, 21-23 March 2019; IEEE: Piscataway, NJ, USA, 2019; pp. 561-566.

63. Arefin, M.T.; Ali, M.H.; Haque, A.F. Wireless body area network: An overview and various applications. J. Comput. Commun. 2017, 5, 53-64. [CrossRef]

64. Vallejos de Schatz, C.H.; Medeiros, H.P.; Schneider, F.K.; Abatti, P.J. Wireless medical sensor networks: Design requirements and enabling technologies. Telemed. E-Health 2012, 18, 394-399. [CrossRef]

65. Reich, O.; Hübner, E.; Ghita, B.; Wagner, M.; Schäfer, J. Performance Evaluation of Bluetooth in a Wireless Body Area Network for Practical Applications. In Proceedings of the 2020 IEEE 11th Sensor Array and Multichannel Signal Processing Workshop (SAM), Hangzhou, China, 8-11 June 2020; pp. 1-5. [CrossRef]

66. Georgakakis, E.; Nikolidakis, S.A.; Vergados, D.D.; Douligeris, C. An analysis of bluetooth, zigbee and bluetooth low energy and their use in wbans. In International Conference on Wireless Mobile Communication and Healthcare; Springer: Berlin/Heidelberg, Germany, 2010; pp. 168-175.

67. Coskun, V.; Ozdenizci, B.; Ok, K. A survey on near field communication (NFC) technology. Wirel. Pers. Commun. 2013, 71, 2259-2294. [CrossRef]

68. Bouhassoune, I.; Saadane, R.; Chehri, A. Wireless Body Area Network Based on RFID System for Healthcare Monitoring: Progress and Architectures. In Proceedings of the 2019 15th International Conference on Signal-Image Technology Internet-Based Systems (SITIS), Sorrento, Italy, 26-29 November 2019; pp. 416-421. [CrossRef]

69. Liu, H.; Bolic, M.; Nayak, A.; Stojmenovi, I. Integration of RFID and wireless sensor networks. In Encyclopedia on Ad Hoc and Ubiquitous Computing: Theory and Design of Wireless Ad Hoc, Sensor, and Mesh Networks; World Scientific: Singapore, 2010; pp. 319-347.

70. Tang, M.; Jin, Y.; Yao, L. WiFi-ZigBee Coexistence Based on Collision Avoidance for Wireless Body Area Network. In Proceedings of the 2017 3rd International Conference on Big Data Computing and Communications (BIGCOM), Chengdu, China, 10-11 August 2017; pp. 245-250. [CrossRef]

71. Casals, L.; Mir, B.; Vidal, R.; Gomez, C. Modeling the energy performance of LoRaWAN. Sensors 2017, 17, 2364. [CrossRef]

72. Haxhibeqiri, J.; Van den Abeele, F.; Moerman, I.; Hoebeke, J. LoRa scalability: A simulation model based on interference measurements. Sensors 2017, 17, 1193. [CrossRef]

73. Gomez, C.; Veras, J.C.; Vidal, R.; Casals, L.; Paradells, J. A sigfox energy consumption model. Sensors 2019, 19, 681. [CrossRef]

74. Lavric, A.; Petrariu, A.I.; Popa, V. Long Range SigFox Communication Protocol Scalability Analysis Under Large-Scale, HighDensity Conditions. IEEE Access 2019, 7, 35816-35825. [CrossRef]

75. Lauridsen, M.; Krigslund, R.; Rohr, M.; Madueno, G. An empirical NB-IoT power consumption model for battery lifetime estimation. In Proceedings of the 2018 IEEE 87th Vehicular Technology Conference (VTC Spring), Porto, Portugal, 3-6 June 2018; IEEE: Piscataway, NJ, USA, 2018; pp. 1-5.

76. Migabo, E.M.; Djouani, K.D.; Kurien, A.M. The Narrowband Internet of Things (NB-IoT) Resources Management Performance State of Art, Challenges, and Opportunities. IEEE Access 2020, 8, 97658-97675. [CrossRef]

77. Díaz Zayas, A.; Rivas Tocado, F.J.; Rodríguez, P. Evolution and Testing of NB-IoT Solutions. Appl. Sci. 2020, 10, 7903. [CrossRef]

78. Tan, Y.K.; Panda, S.K. Review of Energy Harvesting Technologies for Sustainable WSN. Sustain. Wirel. Sens. Netw. 2010, 15-43. [CrossRef]

79. Park, D.Y.; Joe, D.J.; Kim, D.H.; Park, H.; Han, J.H.; Jeong, C.K.; Park, H.; Park, J.G.; Joung, B.; Lee, K.J. Self-powered real-time arterial pulse monitoring using ultrathin epidermal piezoelectric sensors. Adv. Mater. 2017, 29, 1702308. [CrossRef] [PubMed] 
80. Zeng, Y.; Xiang, H.; Zheng, N.; Cao, X.; Wang, N.; Wang, Z.L. Flexible triboelectric nanogenerator for human motion tracking and gesture recognition. Nano Energy 2022, 91, 106601. [CrossRef]

81. Zhu, M.; Sun, Z.; Chen, T.; Lee, C. Low cost exoskeleton manipulator using bidirectional triboelectric sensors enhanced multiple degree of freedom sensory system. Nat. Commun. 2021, 12, 2692. [CrossRef]

82. Bian, S.; Zhu, B.; Rong, G.; Sawan, M. Towards wearable and implantable continuous drug monitoring: A review. J. Pharm. Anal. 2021, 11, 1-14. [CrossRef]

83. Zou, Y.; Bo, L.; Li, Z. Recent progress in human body energy harvesting for smart bioelectronic system. Fundam. Res. 2021, 1, 364-382. [CrossRef]

84. Liu, L.; Guo, X.; Liu, W.; Lee, C. Recent Progress in the Energy Harvesting Technology-From Self-Powered Sensors to Self-Sustained IoT, and New Applications. Nanomaterials 2021, 11, 2975. [CrossRef]

85. Jiang, D.; Shi, B.; Ouyang, H.; Fan, Y.; Wang, Z.L.; Li, Z. Emerging implantable energy harvesters and self-powered implantable medical electronics. ACS Nano 2020, 14, 6436-6448. [CrossRef] [PubMed]

86. Zhao, Y.; Luo, X. Task Offloading Policy for Nodes with Energy Harvesting Capabilities. In Proceedings of the 2019 IEEE 90 th Vehicular Technology Conference (VTC2019-Fall), Honolulu, HI, USA, 22-25 September 2019; pp. 1-5. [CrossRef]

87. Zhang, Y.; He, J.; Guo, S. Energy-Efficient Dynamic Task Offloading for Energy Harvesting Mobile Cloud Computing. In Proceedings of the 2018 IEEE International Conference on Networking, Architecture and Storage (NAS), Chongqing, China, 11-14 October 2018; pp. 1-4. [CrossRef]

88. Guo, M.; Li, Q.; Peng, Z.; Liu, X.; Cui, D. Energy harvesting computation offloading game towards minimizing delay for mobile edge computing. Comput. Netw. 2021, 204, 108678. [CrossRef]

89. Sun, Y.; Song, C.; Yu, S.; Liu, Y.; Pan, H.; Zeng, P. Energy-Efficient Task Offloading Based on Differential Evolution in Edge Computing System with Energy Harvesting. IEEE Access 2021, 9, 16383-16391. [CrossRef]

90. Min, M.; Xiao, L.; Chen, Y.; Cheng, P.; Wu, D.; Zhuang, W. Learning-based computation offloading for IoT devices with energy harvesting. IEEE Trans. Veh. Technol. 2019, 68, 1930-1941. [CrossRef]

91. Khan, P.W.; Abbas, K.; Shaiba, H.; Muthanna, A.; Abuarqoub, A.; Khayyat, M. Energy efficient computation offloading mechanism in multi-server mobile edge computing-An integer linear optimization approach. Electronics 2020, 9, 1010. [CrossRef]

92. Kawata, M.; Takakura, A. Thermoelectrically Powered Wrist Watch. US Patent 5,889,735, 30 March 1999.

93. Hoang, D.C.; Tan, Y.K.; Chng, H.B.; Panda, S.K. Thermal energy harvesting from human warmth for wireless body area network in medical healthcare system. In Proceedings of the 2009 International conference on power electronics and drive systems (PEDS), Taipei, Taiwan, 2-5 November 2009; IEEE: Piscataway, NJ, USA, 2009; pp. 1277-1282.

94. Jouhara, H.; Żabnieńska-Góra, A.; Khordehgah, N.; Doraghi, Q.; Ahmad, L.; Norman, L.; Axcell, B.; Wrobel, L.; Dai, S. Thermoelectric generator (TEG) technologies and applications. Int. J. Thermofluids 2021, 9, 100063. [CrossRef]

95. Halim, M.A.; Cho, H.; Park, J.Y. Design and experiment of a human-limb driven, frequency up-converted electromagnetic energy harvester. Energy Convers. Manag. 2015, 106, 393-404. [CrossRef]

96. Surangsrirat, D.; Dumnin, S.; Samphanyuth, S. Heart Rate, Skin Temperature and Skin Humidity and their Relationship to Accumulated Fatigue. In Proceedings of the 2019 3rd International Conference on Bio-engineering for Smart Technologies (BioSMART), Paris, France, 24-26 April 2019; IEEE: Piscataway, NJ, USA, 2019; pp. 1-4.

97. Kirstein, K.U.; Sedivy, J.; Salo, T.; Hagleitner, C.; Vancura, T.; Baltes, H. A CMOS-based tactile sensor for continuous blood pressure monitoring. In Proceedings of the 30th European Solid-State Circuits Conference, Leuven, Belgium, 23 September 2004; IEEE: Piscataway, NJ, USA, 2004; pp. 463-466.

98. Xiao, Z.; Tan, X.; Chen, X.; Chen, S.; Zhang, Z.; Zhang, H.; Wang, J.; Huang, Y.; Zhang, P.; Zheng, L.; et al. An implantable RFID sensor tag toward continuous glucose monitoring. IEEE J. Biomed. Health Inform. 2015, 19, 910-919. [CrossRef]

99. Shruthi, P.; Resmi, R. Heart rate monitoring using pulse oximetry and development of fitness application. In Proceedings of the 2019 2nd International Conference on Intelligent Computing, Instrumentation and Control Technologies (ICICICT), Kannur India, 5-6 July 2019; IEEE: Piscataway, NJ, USA, 2019; Volume 1, pp. 1568-1570.

100. Ammar, M.B.; Sahnoun, S.; Fakhfakh, A.; Kanoun, O. Design of a DC-DC Boost Converter of Hybrid Energy Harvester for IoT Devices. In Proceedings of the 2020 IEEE 6th World Forum on Internet of Things (WF-IoT), New Orleans, LA, USA, 2-16 June 2020; IEEE: Piscataway, NJ, USA, 2020; pp. 1-4.

101. Ammar, M.B.; Bouattour, G.; Bouhamed, A.; Sahnoun, S.; Fakhfakh, A.; Kanoun, O. AC-DC Single Phase Rectifiers for Nanocomposite based Flexible Piezoelectric Energy Harvesters. In Proceedings of the 2021 18th International Multi-Conference on Systems, Signals \& Devices (SSD), Monastir, Tunisia, 22-25 March 2021; IEEE: Piscataway, NJ, USA, 2021; pp. 228-234.

102. Zouari, M.; Naifar, S.; Götz, M.; Derbel, N.; Kanoun, O. An optimized self-powered P-SSHI circuit for piezoelectric energy harvesting. In Proceedings of the 2017 IEEE International Instrumentation and Measurement Technology Conference (I2MTC), Turin, Italy, 22-25 May 2017; IEEE: Piscataway, NJ, USA, 2017, pp. 1-6.

103. Kanoun, O.; Bradai, S.; Khriji, S.; Bouattour, G.; El Houssaini, D.; Ben Ammar, M.; Naifar, S.; Bouhamed, A.; Derbel, F.; Viehweger, C. Energy-aware system design for autonomous wireless sensor nodes: A comprehensive review. Sensors 2021, 21, 548. [CrossRef]

104. Mallela, V.S.; Ilankumaran, V.; Rao, N.S. Trends in cardiac pacemaker batteries. Indian Pacing Electrophysiol. J. $2004,4,201$.

105. Khalid, S.; Raouf, I.; Khan, A.; Kim, N.; Kim, H.S. A review of human-powered energy harvesting for smart electronics: Recent progress and challenges. Int. J. Precis. Eng. Manuf.-Green Technol. 2019, 6, 821-851. [CrossRef] 
106. Zhou, M.; Al-Furjan, M.S.H.; Zou, J.; Liu, W. A review on heat and mechanical energy harvesting from human-Principles, prototypes and perspectives. Renew. Sustain. Energy Rev. 2018, 82, 3582-3609. [CrossRef]

107. von Buren, T.; Mitcheson, P.; Green, T.; Yeatman, E.; Holmes, A.; Troster, G. Optimization of inertial micropower Generators for human walking motion. IEEE Sensors J. 2006, 6, 28-38. [CrossRef]

108. Yuan, J.; Zhu, R. Self-Powered Wearable Multi-Sensing Bracelet with Flexible Thermoelectric Power Generator. In Proceedings of the 2019 20th International Conference on Solid-State Sensors, Actuators and Microsystems \& Eurosensors XXXIII (TRANSDUCERS \& EUROSENSORS XXXIII), Berlin, Germany, 23-27 June 2019; IEEE: Piscataway, NJ, USA, 2019; pp. 1431-1434.

109. Nozariasbmarz, A.; Collins, H.; Dsouza, K.; Polash, M.H.; Hosseini, M.; Hyland, M.; Liu, J.; Malhotra, A.; Ortiz, F.M.; Mohaddes, F.; et al. Review of wearable thermoelectric energy harvesting: From body temperature to electronic systems. Appl. Energy 2020, 258, 114069. [CrossRef]

110. Zurbuchen, A.; Pfenniger, A.; Stahel, A.; Stoeck, C.T.; Vandenberghe, S.; Koch, V.M.; Vogel, R. Energy harvesting from the beating heart by a mass imbalance oscillation generator. Ann. Biomed. Eng. 2013, 41, 131-141. [CrossRef]

111. Magno, M.; Boyle, D. Wearable energy harvesting: From body to battery. In Proceedings of the 201712 th International Conference on Design \& Technology of Integrated Systems In Nanoscale Era (DTIS), Palma de Mallorca, Spain, 4-6 April 2017; IEEE: Piscataway, NJ, USA, 2017; pp. 1-6.

112. Bhatia, D.; Jo, S.H.; Ryu, Y.; Kim, Y.; Kim, D.H.; Park, H.S. Wearable triboelectric nanogenerator based exercise system for upper limb rehabilitation post neurological injuries. Nano Energy 2021, 80, 105508. [CrossRef]

113. Health Sector Transformation Strategy. V.3. 2019. Available online: https://www.moh.gov.sa/en/Ministry/vro/Documents/ Healthcare-Transformation-Strategy.pdf (accessed on 10 January 2022).

114. Walraven, J.; Jacobs, M.S.; Uyl-de Groot, C.A. Leveraging the Similarities Between Cost-Effectiveness Analysis and Value-Based Healthcare. Value Health 2021, 24, 1038-1044. [CrossRef]

115. Wehde, M. Healthcare 4.0. IEEE Eng. Manag. Rev. 2019, 47, 24-28. [CrossRef]

116. Tirkel, T.; Edan, Y.; Khvorostianov, N.; Bar-Haim, S. SIT LESS: A prototype home-based system for monitoring older adults sedentary behavior. Assist. Technol. 2020, 32, 79-91. [CrossRef]

117. Peng, G.; Sepulveda Garcia, L.M.; Nunes, M.; Zhang, N. Identifying user requirements of wearable healthcare technologies for Chinese ageing population. In Proceedings of the 2016 IEEE International Smart Cities Conference (ISC2), Trento, Italy, 12-15 September 2016; pp. 1-6. [CrossRef] 